\title{
Asymptotic analysis of the Krawtchouk polynomials by the WKB method
}

\author{
Diego Dominici * \\ Department of Mathematics \\ State University of New York at New Paltz \\ 75 S. Manheim Blvd. Suite 9 \\ New Paltz, NY 12561-2443 \\ USA
}

August 28, 2018

\begin{abstract}
We analyze the Krawtchouk polynomials $K_{n}(x, N, p, q)$ asymptotically. We use singular perturbation methods to analyze them for $N \rightarrow \infty$, with appropriate scalings of the two variables $x$ and $n$. In particular, the WKB method and asymptotic matching are used. We obtain asymptotic approximations valid in the whole domain $[0, N] \times$ $[0, N]$, involving some special functions. We give numerical examples showing the accuracy of our formulas.
\end{abstract}

\section{Introduction}

Definition 1 Let $n, N \geq 0$ be integers. The Krawtchouk polynomials $K_{n}(x)$ are defined by [36],

$$
K_{n}(x)=\sum_{k=0}^{n}\left(\begin{array}{l}
x \\
k
\end{array}\right)\left(\begin{array}{c}
N-x \\
n-k
\end{array}\right) q^{k}(-p)^{n-k}
$$

where

$$
0<p, q<1 \quad p+q=1 .
$$

The binary Krawtchouk polynomials are the special case with $p=1 / 2=q$ [15], [18].

*e-mail: dominicd@newpaltz.edu 
The Krawtchouk polynomials [27] are one of the families of classical orthogonal polynomials of a discrete variable [14]. They satisfy the orthogonality relation

$$
\sum_{k=0}^{N} K_{i}(k) K_{j}(k) \varrho(k)=\left(\begin{array}{c}
N \\
j
\end{array}\right)(p q)^{j} \delta_{i j}, \quad i, j=0, \ldots, N
$$

with weight function

$$
\varrho(x)=\left(\begin{array}{c}
N \\
x
\end{array}\right) p^{x} q^{N-x} .
$$

Writing the Krawtchouk polynomials in the extended form $K_{n}(x)=K_{n}(x, N, p, q)$, we have the the symmetry formula

$$
K_{n}(x, N, p, q)=(-1)^{n} K_{n}(N-x, N, q, p) .
$$

They also satisfy the three-term recurrence

$$
(n+1) K_{n+1}(x)+p q(N-n+1) K_{n-1}(x)+[p(N-n)+n q-x] K_{n}(x)=0
$$

which is the main object of our analysis.

The Krawtchouk polynomials are important in the study of the Hamming scheme of classical coding theory [17, 21], 25], 29], 33, 34]. Lloyd's theorem [23] states that if a perfect code exists in the Hamming metric, then the Krawtchouk polynomial must have integral zeros [3], [5], [20]. Not surprisingly, these zeros have been the subject of extensive research 4], 7] [9], 10], 11], [16], [35].

The Krawtchouk polynomials also have applications in probability theory [8], queueing models [6], stochastic processes [31], quantum mechanics [2], 24] [39] and biology [13].

The asymptotic behavior of the Krawtchouk polynomials as $N \rightarrow \infty$ was studied by Sharapudinov for $x \approx N p$ and $n=O\left(N^{1 / 3}\right)$ in 32. He derived an approximation in terms of the Hermite polynomials (see also [36] for a similar formula). The general case with $x, n=O(N)$ was investigated by Ismail and Simeonov [12] and a uniform asymptotic expansion was derived by Li and Wong [22], both using the saddle point method.

The purpose of this paper is to take a different approach, based on the recurrence formula that the Krawtchouk polynomials satisfy and using singular perturbation techniques [26], [37] to analyze it. We scale $x=y N, n=z N$ and obtain asymptotic approximations to $K_{n}(x)$ for $(y, z) \in[0,1] \times[0,1]$. Our results agree and extend those obtained in [12, 22] and 30].

In Section 2 we review the basic properties of the Krawtchouk polynomials. In Sections 310 we obtain asymptotic expansions from the recurrence formula by using the WKB method. We must consider twelve relevant regions of the two-dimensional state space. In Section 11 we summarize our results and numerically compare our approximations with the exact formula. 


\section{The WKB approximation}

To analyze the recurrence

$$
(n+1) K_{n+1}(x)+p q(N-n+1) K_{n-1}(x)+[p(N-n)+n q-x] K_{n}(x)=0
$$

subject to the boundary conditions

$$
\begin{gathered}
K_{0}(x)=1 \\
K_{N+1}(x)=\left(\begin{array}{c}
x \\
N+1
\end{array}\right) \\
K_{n}(0)=\left(\begin{array}{c}
N \\
n
\end{array}\right)(-p)^{n} \\
K_{n}(N)=\left(\begin{array}{c}
N \\
n
\end{array}\right) q^{n}
\end{gathered}
$$

for large $N$, we introduce the scaled variables $y, z$ defined by

$$
x=y N, \quad n=z N, \quad 0<y, z<1 .
$$

We define the function $F(y, z)$ and the small parameter $\varepsilon$ by

$$
\varepsilon=\frac{1}{N}, \quad K_{n}(x)=F(\varepsilon x, \varepsilon n)=F(y, z)
$$

and observe that $K_{n \pm 1}(x)=F(y, z \pm \varepsilon)$.

Substituting (9)-(10) in (4) we get

$$
(z+\varepsilon) F(y, z+\varepsilon)+p q(1-z+\varepsilon) F(y, z-\varepsilon)+[p(1-z)+z q-y] F(y, z)=0 .
$$

To find $F(y, z)$ for $\varepsilon$ small, we shall use the WKB method [28]. Thus, we consider solutions which have the asymptotic form

$$
F(y, z) \sim \varepsilon^{\nu} \exp \left[\varepsilon^{-1} \psi(y, z)\right] L(y, z) .
$$

Using (12) in (11), with

$$
\varepsilon^{-1} \psi(y, z \pm \varepsilon)=\varepsilon^{-1} \psi(y, z) \pm \psi_{z}(y, z)+\frac{1}{2} \psi_{z z}(y, z) \varepsilon+O\left(\varepsilon^{2}\right)
$$

dividing by $\exp \left[\varepsilon^{-1} \psi(y, z)\right]$ and expanding in powers of $\varepsilon$ we obtain the eikonal equation

$$
z U^{2}+[p-y+z(q-p)] U+p q(1-z)=0
$$

and the transport equation

$$
\left[z U^{2}-p q(1-z)\right] L_{z}+\left\{\frac{1}{2}\left[z U^{2}+p q(1-z)\right] \psi_{z z}+U^{2}+p q\right\} L=0
$$

with

$$
U(y, z)=\exp \left[\psi_{z}(y, z)\right]
$$




\subsection{The functions $\psi$ and $L$}

From (15) we have

$$
\psi(y, z)=\int \ln [U(y, z)] d z=z \ln (U)-\int z \frac{U_{z}}{U} d z=z \ln (U)-\int z \frac{U_{z}}{U} \frac{d z}{d U} d U
$$

and using (13) we get

$$
\int z \frac{U_{z}}{U} \frac{d z}{d U} d U=\int \frac{(y-p) U-p q}{(U+q)(U-p)} d U=\int\left[\frac{1}{U}+\frac{y-1}{U}-\frac{y}{U+q}\right] d U
$$

Hence,

$$
\psi(y, z)=\ln \left[U^{z-1}(U-p)^{1-y}(U+q)^{y}\right]+A(y)
$$

where the function $A(y)$ is still unknown.

From (14) we have

$$
L(y, z)=B(y) \exp \left[-\int \frac{\frac{1}{2}\left[z U^{2}+p q(1-z)\right] \psi_{z z}+U^{2}+p q}{z U^{2}-p q(1-z)} d z\right]
$$

and from (15) $\psi_{z z}=U_{z} / U$. After changing variables from $z$ to $U$, we obtain

$$
L(y, z)=B(y) \exp \left\{\int\left[\frac{1}{2 U}+\frac{1}{U+q}-\frac{(p-y) U+p q}{(p-y) U^{2}+2 p q U+p q(q-y)}\right] d U\right\} .
$$

Thus,

$$
L(y, z)=B(y)(U+q) \sqrt{\frac{U}{(p-y) U^{2}+2 p q U+p q(q-y)}}
$$

where $B(y)$ is to be determined.

\subsection{The function $U$}

Rewriting (13) as

$$
U^{2}+\left(\frac{p-y}{z}+q-p\right) U+\left(U_{0}\right)^{2}=0
$$

and solving for $U$ we get

$$
U^{ \pm}(y, z)=-\frac{1}{2}\left(\frac{p-y}{z}+q-p\right) \pm \frac{1}{2} \sqrt{\left(\frac{p-y}{z}+q-p\right)^{2}-4\left(U_{0}\right)^{2}}
$$

where

$$
U_{0}(z)=\sqrt{\frac{p q(1-z)}{z}}
$$


The discriminant in (18) vanishes if $\frac{p-y}{z}+q-p= \pm 2 U_{0}$, which is equivalent to $y=Y^{ \pm}$, with

$$
Y^{ \pm}(z)=p+(q-p) z \pm 2 z U_{0}
$$

Rewriting the equation $\left(\frac{p-y}{z}+q-p\right)^{2}-4\left(U_{0}\right)^{2}=0$ as

$$
\left(y-\frac{1}{2}\right)^{2}+\left(z-\frac{1}{2}\right)^{2}+2(p-q)\left(y-\frac{1}{2}\right)\left(z-\frac{1}{2}\right)-p q=0
$$

we can see that

$$
\left\{(y, z) \in[0,1] \times[0,1]: y=Y^{ \pm}(z)\right\}=\mathbb{E}
$$

where $\mathbb{E}$ is an ellipse centered at $\left(\frac{1}{2}, \frac{1}{2}\right)$ (see Figure 1). After rotation by $\pm \frac{\pi}{4}$ and translation to the origin, $\mathbb{E}$ reduces to one of the canonical forms

$$
\begin{aligned}
\frac{\bar{y}^{2}}{q / 2}+\frac{\bar{z}^{2}}{p / 2} & =1, & & p<q \\
\bar{y}^{2}+\bar{z}^{2} & =\frac{1}{4}, & & p=\frac{1}{2}=q \\
\frac{\bar{y}^{2}}{p / 2}+\frac{\bar{z}^{2}}{q / 2} & =1, & & p>q .
\end{aligned}
$$

The ellipse $\mathbb{E}$ is contained in the square $[0,1] \times[0,1]$ and intersects the $y$ and $z$ axis at the points $(0, p),(1, q),(p, 0)$ and $(q, 1)$. Its left side between the points $(q, 1)$ and $(p, 0)$ coincides with the curve $Y^{-}(z)$ and its right side with the curve $Y^{+}(z)$.

For points $(y, z)$ located outside $\mathbb{E}, U^{ \pm}(y, z)$ are real and for points $(y, z)$ inside $\mathbb{E} U^{ \pm}(y, z)$ are complex conjugates. When $y=Y^{ \pm}$the two values $U^{ \pm}$coalesce and we have

$$
U^{+}\left(Y^{+}, z\right)=U_{0}=U^{-}\left(Y^{+}, z\right), \quad U^{+}\left(Y^{-}, z\right)=-U_{0}=U^{-}\left(Y^{-}, z\right) .
$$

Writing the function $L(y, z)$ in terms of $U_{0}$ we have from (17)

$$
L(y, z)=B(y) \sqrt{\frac{(U-p)(U+q)}{z\left(U^{2}-U_{0}^{2}\right)}} .
$$

In the rest of this paper we shall use the following notation

$$
\psi^{ \pm}(y, z)=\ln \left[\left(U^{ \pm}\right)^{z-1}\left(U^{ \pm}-p\right)^{1-y}\left(U^{ \pm}+q\right)^{y}\right]
$$

and

$$
L^{ \pm}(y, z)=\sqrt{\frac{\left(U^{ \pm}-p\right)\left(U^{ \pm}+q\right)}{z\left[\left(U^{ \pm}\right)^{2}-U_{0}^{2}\right]}} .
$$

Hence, we write

$$
\begin{aligned}
K_{n}(x) & \sim \varepsilon^{\nu} B^{-}(y) \exp \left[\varepsilon^{-1} \psi^{-}(y, z)+\varepsilon^{-1} A^{-}(y)\right] L^{-}(y, z) \\
& +\varepsilon^{\nu} B^{+}(y) \exp \left[\varepsilon^{-1} \psi^{+}(y, z)+\varepsilon^{-1} A^{+}(y)\right] L^{+}(y, z)
\end{aligned}
$$

where $B^{ \pm}(y)$ and $A^{ \pm}(y)$ are functions to be determined. From (26) we see that $L^{ \pm}(y, z)$ are singular when $U^{ \pm}=U_{0}$, i.e., for $y=Y^{ \pm}$, and also for $z=0$. Therefore, we need to find asymptotic solutions valid in those regions. 


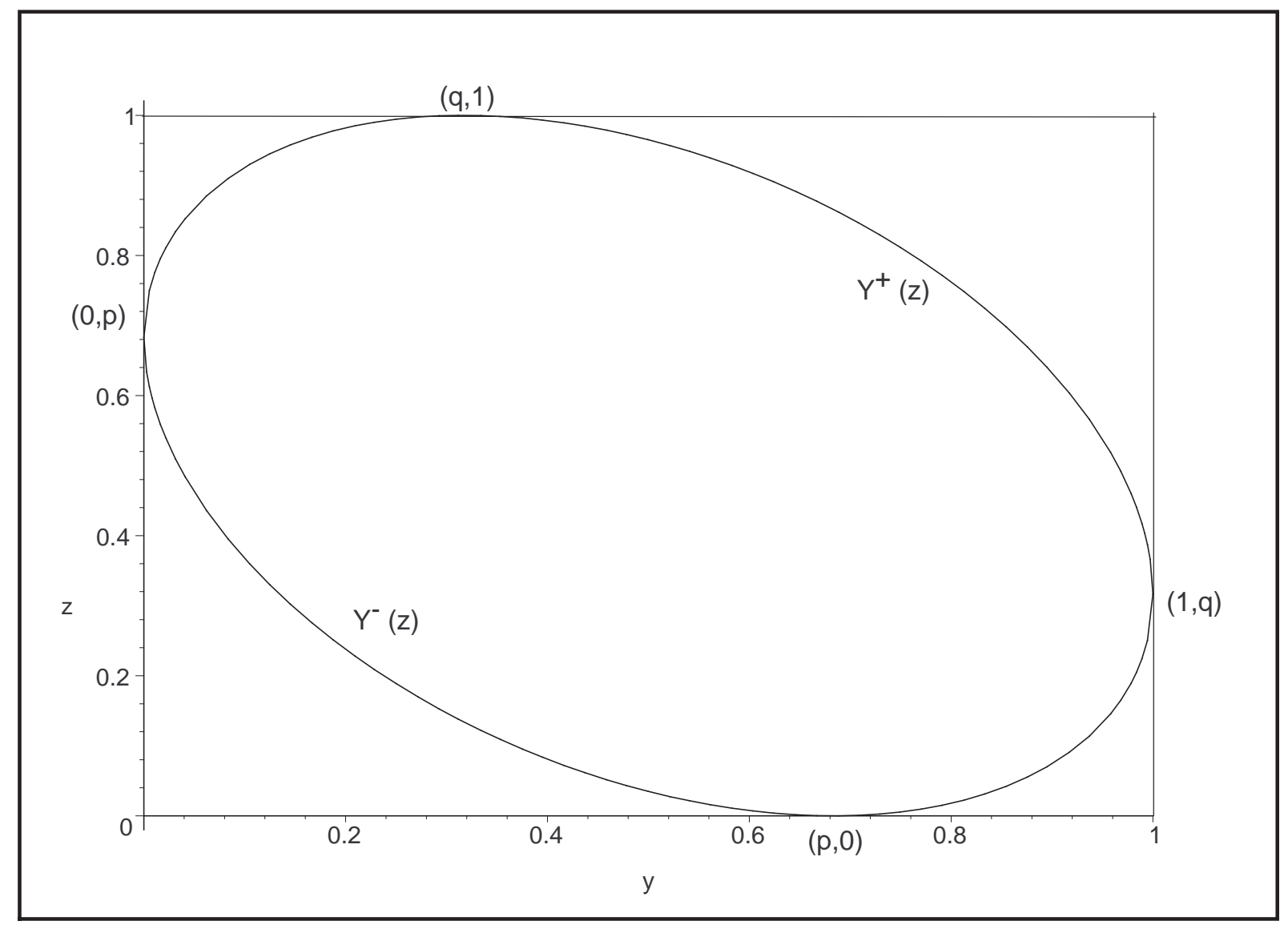

Figure 1: A sketch of the ellipse $\mathbb{E}$ and the curves $Y^{ \pm}(z)$. 


\section{$3 \quad$ The boundary $n=0$ (Region I)}

For $n=O(1)$ we have from (11) that $K_{n}(x)=O\left(N^{n}\right)$ as $N \rightarrow \infty$. Thus, we introduce the function $R_{n}^{(1)}(y)$ and consider solutions of (41) which have the asymptotic form

$$
K_{n}(x)=N^{n} R_{n}^{(1)}\left(\frac{x}{N}\right)
$$

Using (28) in (41) and expanding in powers of $N$ gives, to leading order

$$
(n+1) R_{n+1}^{(1)}+(p-y) R_{n}^{(1)}=0 .
$$

Solving the recursion above with the initial condition (5) we get

$$
R_{n}^{(1)}(y)=\frac{(y-p)^{n}}{n !}
$$

and hence

$$
K_{n}(x) \sim K_{n}^{(1)}(y)=N^{n} \frac{(y-p)^{n}}{n !}, \quad n=O(1)
$$

\subsection{The corner layer at $(p, 0)$ (Region II)}

We shall now find an asymptotic solution in the neighborhood of the point $(p, 0)$. We introduce the stretched variable $\eta$ and the function $R_{n}^{(2)}(\eta)$ defined by

$$
\begin{aligned}
y & =p+\eta \sqrt{2 p q \varepsilon} \quad \eta=O(1), \\
K_{n}(x) & =\frac{\varepsilon^{-n / 2}}{n !}\left(\frac{p q}{2}\right)^{n / 2} R_{n}^{(2)}\left(\frac{\varepsilon x-p}{\sqrt{2 p q \varepsilon}}\right) .
\end{aligned}
$$

Using (30) in (4) yields, to leading order, the equation

$$
R_{n+1}^{(2)}-2 \eta R_{n}^{(2)}+2 n R_{n-1}^{(2)}=0
$$

which we recognize as the recurrence relation for the Hermite polynomials. Thus,

$$
K_{n}(x) \sim K_{n}^{(2)}(\eta)=\frac{\varepsilon^{-n / 2}}{n !}\left(\frac{p q}{2}\right)^{n / 2} H_{n}(\eta)
$$

for $n=O(1)$ and $y-p=O\left(\varepsilon^{1 / 2}\right)$, where $H_{n}(\eta)$ is the Hermite polynomial of degree $n$.

\section{The lower corners (Regions III and IV)}

Setting $n=z / \varepsilon$ in (29) and letting $\varepsilon \rightarrow 0$ we obtain

$$
K_{n}^{(1)}(y) \sim \varepsilon^{1 / 2} \frac{1}{\sqrt{2 \pi z}} \exp \left\{\varepsilon^{-1}[1-\ln (z)+\ln (y-p)] z\right\}
$$


where we have used Stirling's formula [1]

$$
\Gamma(x) \sim \sqrt{\frac{2 \pi}{x}} x^{x} e^{-x}, \quad x \rightarrow \infty .
$$

From (25)-(26) we have as $z \rightarrow 0$

$$
\begin{aligned}
& \psi^{-}(y, z) \sim\left\{\begin{array}{c}
{[1-\ln (z)+\ln (y-p)] z, \quad y<p} \\
(1-y) \ln \left(\frac{1-y}{q}\right)+y \ln \left(\frac{y}{p}\right)+\ln \left(\frac{p q}{y-p}\right) z, \quad y>p
\end{array}\right. \\
& L^{-}(y, z)=\left\{\begin{array}{cc}
z^{-1 / 2}+O(z), & y<p \\
\frac{\sqrt{(y-1) y}}{y-p}+O(z), & y>p
\end{array}\right. \\
& \psi^{+}(y, z) \sim\left\{\begin{array}{c}
(1-y) \ln \left(\frac{1-y}{q}\right)+y \ln \left(\frac{y}{p}\right)+\ln \left(\frac{p q}{y-p}\right) z, \quad y<p \\
{[1-\ln (z)+\ln (y-p)] z, \quad y>p}
\end{array}\right.
\end{aligned}
$$

and

$$
L^{+}(y, z)=\left\{\begin{array}{cc}
\frac{\sqrt{(y-1) y}}{p-y}+O(z), & y<p \\
z^{-1 / 2}+O(z), & y>p
\end{array} .\right.
$$

Matching (34)-(37) and (32) we conclude that

$$
K_{n}(x) \sim \begin{cases}K^{-}(y, z), & 0<y<Y^{-}(z) \\ K^{+}(y, z), & Y^{+}(z)<y<1\end{cases}
$$

with

$$
\begin{aligned}
& K^{-}(y, z)=\varepsilon^{1 / 2} \frac{1}{\sqrt{2 \pi}} \exp \left[\varepsilon^{-1} \psi^{-}(y, z)\right] L^{-}(y, z) \\
& K^{+}(y, z)=\varepsilon^{1 / 2} \frac{1}{\sqrt{2 \pi}} \exp \left[\varepsilon^{-1} \psi^{+}(y, z)\right] L^{+}(y, z) .
\end{aligned}
$$

Remark 2 In the remainder of the paper, we will find asymptotic formulas only in the region $0 \leq y \leq Y^{+}(z), 0 \leq z \leq 1$. The corresponding results for $Y^{+}(z) \leq y \leq 1$ can be obtained by using the symmetry formula (3) and noting that under the transformations $y \rightarrow 1-y$, $p \leftrightarrow q, K_{n} \rightarrow(-1)^{n} K_{n}$, we obtain

$$
\begin{aligned}
U^{-} & \rightarrow U^{+} \\
\psi^{-} & \rightarrow \psi^{+}+z \pi \mathrm{i}, \quad L^{-} \rightarrow L^{+} \\
K^{-} & \rightarrow K^{+} .
\end{aligned}
$$




\section{The boundary $x=0$}

We shall now consider the case $x=O(1)$.

Lemma 3 Let $x=m$, with $m$ an integer, $m \ll N$.

1.

$$
K_{n}(m)=(-p)^{n}\left(\begin{array}{c}
N \\
n
\end{array}\right)\left(1-\frac{n}{p} N^{-1}\right)^{m}, \quad m=0,1 .
$$

2. If $n=O(N)$, then

$$
K_{n}(m) \sim(-p)^{n}\left(\begin{array}{c}
N \\
n
\end{array}\right)\left(1-\frac{n}{p} N^{-1}\right)^{m}, \quad N \rightarrow \infty, \quad m \geq 2 .
$$

Proof. From (11) we have for $x=m$ integer

$$
\begin{aligned}
K_{n}(m) & =\sum_{k=0}^{m}\left(\begin{array}{c}
m \\
k
\end{array}\right)\left(\begin{array}{c}
N-m \\
n-k
\end{array}\right) q^{k}(-p)^{n-k} \\
& =(-p)^{n}\left(\begin{array}{c}
N \\
n
\end{array}\right) \sum_{k=0}^{m}\left(\begin{array}{c}
m \\
k
\end{array}\right) \frac{\left(\begin{array}{c}
N-m \\
n-k
\end{array}\right)}{\left(\begin{array}{l}
N \\
n
\end{array}\right)}\left(-\frac{q}{p}\right)^{k}
\end{aligned}
$$

and (41) follows for $m=0,1$.

Setting $n=z N$ and using (33) we get

$$
\frac{\left(\begin{array}{c}
N-m \\
N z-k
\end{array}\right)}{\left(\begin{array}{c}
N \\
N z
\end{array}\right)} \sim(1-z)^{m}\left(\frac{z}{1-z}\right)^{k}, \quad N \rightarrow \infty .
$$

Using the above in (43) we have

$$
\begin{aligned}
K_{n}(m) & \sim(-p)^{n}\left(\begin{array}{c}
N \\
n
\end{array}\right)(1-z)^{m} \sum_{k=0}^{m}\left(\begin{array}{c}
m \\
k
\end{array}\right)\left(-\frac{z}{1-z} \frac{q}{p}\right)^{k} \\
& =(-p)^{n}\left(\begin{array}{c}
N \\
n
\end{array}\right)(1-z)^{m}\left[\frac{p-z}{p(1-z)}\right]^{m}
\end{aligned}
$$

and (42) follows.

Using (33) we have, as $N \rightarrow \infty$

$$
(-p)^{n}\left(\begin{array}{c}
N \\
n
\end{array}\right) \sim \frac{\varepsilon^{1 / 2}}{\sqrt{2 \pi} \sqrt{z(1-z)}} \exp \left[\varepsilon^{-1} \phi_{0}(z)\right]
$$

where

$$
\phi_{0}(z)=(z-1) \ln (1-z)-z \ln (z)+z \ln (-p) .
$$


From (39) we get, as $y \rightarrow 0$

$$
K^{-}(y, z) \sim \frac{\varepsilon^{1 / 2}}{\sqrt{2 \pi} \sqrt{z(1-z)}} \exp \left[\frac{\phi_{0}(z)+\ln \left(\frac{p-z}{p}\right) y}{\varepsilon}\right], \quad 0<z<p .
$$

Hence, as $y \rightarrow 0, K^{-}(y, z)$ satisfies the boundary condition (77) for $0<z<p$.

\subsection{The boundary layer at $x=0, p<z<1$ (Region V)}

Taking (42) into account, we define the function $R_{n}^{(5)}(x)$ by

$$
K_{n}(x)=\left(\begin{array}{c}
N \\
n
\end{array}\right)(-p)^{n} R_{n}^{(5)}(x) .
$$

Using (47) in (41) yields

$$
p(n-N) R_{n+1}^{(5)}+[p N+(q-p) n-x] R_{n}^{(5)}-n q R_{n-1}^{(5)}=0 .
$$

Writing (48) in terms of $z, \varepsilon$ and the function $G^{(5)}(x, z)$ defined by

$$
R_{n}^{(5)}(x)=G^{(5)}(x, \varepsilon n)
$$

we have

$$
p(1-z) G^{(5)}(x, z+\varepsilon)+[z(p-q)+x \varepsilon-p] G^{(5)}(x, z)+z q G^{(5)}(x, z-\varepsilon)=0 .
$$

Using the WKB anszat

$$
G^{(5)}(x, z) \sim \varepsilon^{\tau} \exp \left[\frac{1}{\varepsilon} \phi(x, z)\right] W(x, z)
$$

in (50), we obtain the equations

$$
\left[\exp \left(\phi_{z}\right)-1\right]\left[p(z-1) \exp \left(\phi_{z}\right)+z q\right] W=0
$$

and

$$
\begin{gathered}
{\left[z q+p(z-1) \exp \left(2 \phi_{z}\right)\right] W_{z}} \\
+\left\{\frac{1}{2}\left[p(z-1) \exp \left(2 \phi_{z}\right)-z q\right] \phi_{z z}-x \exp \left(\phi_{z}\right)\right\} W=0 .
\end{gathered}
$$

Solving (52)-(1531) we get

$$
\begin{aligned}
G^{(5)}(x, z) \sim \varepsilon^{\tau_{1}} A^{(5)}(x) \exp \left[\varepsilon^{-1} \varphi_{1}(x)\right](z-p)^{x} \\
+\varepsilon^{\tau_{2}} B^{(5)}(x) \exp \left[\varepsilon^{-1} \phi_{2}(x, z)\right](z-p)^{-x-1} \sqrt{z(1-z)}
\end{aligned}
$$


where

$$
\phi_{2}(x, z)=(1-z) \ln (1-z)+z \ln (z)+(z-1) \ln (q)-z \ln (p)+\varphi_{2}(x)
$$

and the coefficients $\tau_{1}, \tau_{2}$ and the functions $A^{(5)}(x), B^{(5)}(x), \varphi_{1}(x), \varphi_{2}(x)$ are to be determined.

Using (44) we obtain

$$
\begin{aligned}
K_{n}(x) & \sim K^{(5)}(x, z)=\frac{\varepsilon^{1 / 2+\tau_{1}}}{\sqrt{2 \pi} \sqrt{z(1-z)}} \exp \left[\frac{\phi_{0}(z)+\varphi_{1}(x)}{\varepsilon}\right] A(x)(z-p)^{x} \\
+ & \frac{\varepsilon^{1 / 2+\tau_{2}}}{\sqrt{2 \pi}} \exp \left[\frac{(z-1) \ln (q)+\pi \mathrm{i} z+\varphi_{2}(x)}{\varepsilon}\right] B(x)(z-p)^{-x-1} .
\end{aligned}
$$

\subsection{The corner layer at $(0, p)$ (Region VI)}

For $x \approx 0$ and $n \approx N p$, we scale $n$ as

$$
n=N p-u \sqrt{p q N}, \quad u=O(1)
$$

and introduce the function $G^{(6)}(x, u)$ defined by

$$
K_{n}(x)=\left(\begin{array}{c}
N \\
n
\end{array}\right)(-p)^{n} G^{(6)}\left(x, \frac{N p-n}{\sqrt{p q N}}\right) .
$$

Using (57)-(58) in (48) we get, to leading order

$$
\frac{\partial^{2} G^{(6)}}{\partial u^{2}}-\frac{\partial G^{(6)}}{\partial u}+x G^{(6)}=0
$$

Solving (59) we obtain

$$
G^{(6)}(x, u)=\exp \left(\frac{u^{2}}{4}\right)\left[A^{(6)}(x) \mathrm{D}_{x}(u)+B^{(6)}(x) \mathrm{D}_{x}(-u)\right]
$$

where $D_{x}(u)$ is the parabolic cylinder function and $A^{(6)}(x), B^{(6)}(x)$ are functions to be determined. Since

$$
u=\frac{N p-n}{\sqrt{p q N}}=\frac{p-z}{\sqrt{p q \varepsilon}},
$$

we note that the limit $u \rightarrow \infty$ corresponds to the matching between regions VI and III, while the limit $u \rightarrow-\infty$ corresponds to the matching between regions VI and V. As $z \rightarrow p$ we have

$$
\begin{gathered}
(-p)^{n}\left(\begin{array}{c}
N \\
n
\end{array}\right) \sim \frac{\sqrt{\varepsilon}}{\sqrt{2 \pi p q}} \exp \left(-\frac{u^{2}}{2}\right) \\
\times \exp \left\{\frac{\pi \mathrm{i} p-q \ln (q)}{\varepsilon}-\frac{u \sqrt{p q}[\pi \mathrm{i}+\ln (q)]}{\sqrt{\varepsilon}}\right\} .
\end{gathered}
$$


Thus,

$$
\begin{aligned}
K_{n}(x) & \sim K^{(6)}(x, u)=\frac{\sqrt{\varepsilon}}{\sqrt{2 \pi p q}}\left[A^{(6)}(x) \mathrm{D}_{x}(u)+B^{(6)}(x) \mathrm{D}_{x}(-u)\right] \\
& \times \exp \left\{\frac{\pi \mathrm{i} p-q \ln (q)}{\varepsilon}-\frac{u \sqrt{p q}[\pi \mathrm{i}+\ln (q)]}{\sqrt{\varepsilon}}-\frac{u^{2}}{4}\right\} .
\end{aligned}
$$

Using (61) in (46) yields

$$
\begin{gathered}
K^{-}(y, z) \sim \frac{\sqrt{\varepsilon}}{\sqrt{2 \pi p q}}\left[\sqrt{\frac{q \varepsilon}{p}} u\right]^{x} \\
\times \exp \left[\frac{\pi \mathrm{i} p-q \ln (q)}{\varepsilon}-\frac{u \sqrt{p q}[\pi \mathrm{i}+\ln (q)]}{\sqrt{\varepsilon}}-\frac{u^{2}}{2}\right] .
\end{gathered}
$$

Using the well known asymptotic approximation 38]

$$
\mathrm{D}_{x}(u) \sim \exp \left(-\frac{u^{2}}{4}\right) u^{x}, \quad u \rightarrow \infty
$$

in (63) and comparing with (64) we conclude that

$$
A^{(6)}(x)=\left[\sqrt{\frac{q \varepsilon}{p}}\right]^{x}
$$

and $B^{(6)}(x)=0$. Therefore,

$$
\begin{gathered}
K^{(6)}(x, u)=\frac{\varepsilon^{1 / 2}}{\sqrt{2 \pi p q}}\left[\sqrt{\frac{q \varepsilon}{p}}\right]^{x} \mathrm{D}_{x}(u) \\
\times \exp \left[\frac{\pi \mathrm{i} p-q \ln (q)}{\varepsilon}-\frac{u \sqrt{p q}[\pi \mathrm{i}+\ln (q)]}{\sqrt{\varepsilon}}-\frac{u^{2}}{4}\right]
\end{gathered}
$$

for $x=O(1), z-p=O\left(\varepsilon^{1 / 2}\right)$.

Using the formula 38

$$
\mathrm{D}_{x}(-u) \sim \exp \left(-\frac{u^{2}}{4}\right) u^{x} \cos (\pi x)-\sqrt{\frac{2}{\pi}} x \Gamma(x) \sin (\pi x) u^{-x-1} \exp \left(\frac{u^{2}}{4}\right), \quad u \rightarrow \infty
$$

in (66) we have

$$
\begin{gathered}
K^{(6)}(x,-u) \sim \frac{\varepsilon^{1 / 2}}{\sqrt{2 \pi p q}}\left[u \sqrt{\frac{q \varepsilon}{p}}\right]^{x} \cos (\pi x) \\
\times \exp \left[\frac{\pi \mathrm{i} p-q \ln (q)}{\varepsilon}-\frac{u \sqrt{p q}[\pi \mathrm{i}+\ln (q)]}{\sqrt{\varepsilon}}-\frac{u^{2}}{2}\right] \\
-\frac{1}{u} \frac{\varepsilon^{1 / 2}}{\pi \sqrt{p q}}\left[\frac{1}{u} \sqrt{\frac{q \varepsilon}{p}}\right]^{x} x \Gamma(x) \sin (\pi x) \\
\times \exp \left[\frac{\pi \mathrm{i} p-q \ln (q)}{\varepsilon}-\frac{u \sqrt{p q}[\pi \mathrm{i}+\ln (q)]}{\sqrt{\varepsilon}}\right], \quad u \rightarrow \infty .
\end{gathered}
$$


Using (61) in (56) gives, as $z \downarrow p$

$$
\begin{gathered}
K^{(5)}(x, z) \sim \frac{\varepsilon^{1 / 2+\tau_{1}}}{\sqrt{2 \pi p q}} A^{(5)}(x)(u \sqrt{p q \varepsilon})^{x} \\
\times \exp \left[\frac{\pi \mathrm{i} p-q \ln (q)+\varphi_{1}(x)}{\varepsilon}-\frac{u \sqrt{p q}[\pi \mathrm{i}+\ln (q)]}{\sqrt{\varepsilon}}-\frac{u^{2}}{2}\right] \\
+\exp \left[\frac{\pi \mathrm{i} p-q \ln (q)+\varphi_{2}(x)}{\varepsilon}-\frac{u \sqrt{p q}[\pi \mathrm{i}+\ln (q)]}{\sqrt{\varepsilon}}\right] \\
\times \frac{1}{u} \frac{\varepsilon^{\tau_{2}}}{\sqrt{2 \pi p q}} B^{(5)}(x)(u \sqrt{p q \varepsilon})^{-x} .
\end{gathered}
$$

Matching (68) and (69) yields $\tau_{1}=0, \varphi_{1}(x)=0, \tau_{2}=\frac{1}{2}, \varphi_{2}(x)=0$ and

$$
\begin{aligned}
& A^{(5)}(x)=p^{-x} \cos (\pi x) \\
& B^{(5)}(x)=-\sqrt{\frac{2}{\pi}} x \Gamma(x) \sin (\pi x)(q \varepsilon)^{x} .
\end{aligned}
$$

Thus,

$$
\begin{gathered}
K^{(5)}(x, z)=\frac{\varepsilon^{1 / 2}}{\sqrt{2 \pi} \sqrt{z(1-z)}} \cos (\pi x)\left(\frac{z-p}{p}\right)^{x} \exp \left[\frac{\phi_{0}(z)}{\varepsilon}\right] \\
-\frac{\varepsilon}{\pi} \frac{x}{z-p} \Gamma(x) \sin (\pi x)\left(\frac{q \varepsilon}{z-p}\right)^{x} \exp \left[\frac{(z-1) \ln (q)+\pi \mathrm{i} z}{\varepsilon}\right]
\end{gathered}
$$

for $x=O(1)$ and $p<z<1$.

From (70) we have, as $x \rightarrow \infty$

$$
\begin{aligned}
& K^{(5)}(x, z) \sim \frac{\varepsilon^{1 / 2}}{\sqrt{2 \pi} \sqrt{z(1-z)}} \cos (\pi x)\left(\frac{z-p}{p}\right)^{x} \exp \left[\frac{\phi_{0}(z)}{\varepsilon}\right] \\
& -\varepsilon \sqrt{\frac{2}{\pi}} \frac{\sqrt{x}}{z-p} \sin (\pi x)\left(\frac{q \varepsilon x}{z-p}\right)^{x} \exp \left[\frac{(z-1) \ln (q)+\pi \mathrm{i} z}{\varepsilon}-x\right] .
\end{aligned}
$$

In terms of $y=x \varepsilon$, (71) reads

$$
\begin{gathered}
K^{(5)}(x, z) \sim \frac{\varepsilon^{1 / 2}}{\sqrt{2 \pi} \sqrt{z(1-z)}} \cos \left(\frac{\pi y}{\varepsilon}\right) \exp \left[\frac{\phi_{0}(z)+y \ln \left(\frac{z-p}{p}\right)}{\varepsilon}\right] \\
-\varepsilon^{1 / 2} \sqrt{\frac{2}{\pi}} \sin \left(\frac{\pi y}{\varepsilon}\right) \frac{\sqrt{y}}{z-p} \exp \left[\frac{(z-1) \ln (q)+\pi \mathrm{i} z+y \ln \left(\frac{q y}{z-p}\right)-y}{\varepsilon}\right] .
\end{gathered}
$$




\section{The left upper corner (Region VII)}

We now consider the region $0 \ll y<Y^{-}(z), p<z<1$. From (25)-(26) we have for $y \rightarrow 0, \quad z>p$

$$
\begin{gathered}
\psi^{+}(y, z) \sim \phi_{0}(z)+y \ln \left(\frac{z-p}{p}\right)-\pi \mathrm{i} y \\
\psi^{-}(y, z) \sim(z-1) \ln (q)+\pi \mathrm{i} z+y \ln \left(\frac{q y}{z-p}\right)-y-\pi \mathrm{i} y,
\end{gathered}
$$

and

$$
L^{+}(y, z) \sim \frac{1}{\sqrt{z(1-z)}}, \quad L^{-}(y, z) \sim \frac{\sqrt{y}}{z-p} \mathrm{i} .
$$

Using (73)-(75) in (27) and matching with (72), we conclude that

$$
K_{n}(x) \sim K^{(7)}(y, z)=\exp \left(\frac{\pi \mathrm{i} y}{\varepsilon}\right)\left[\cos \left(\frac{\pi y}{\varepsilon}\right) K^{+}(y, z)+2 \mathrm{i} \sin \left(\frac{\pi y}{\varepsilon}\right) K^{-}(y, z)\right],
$$

which can be written as

$$
K^{(7)}(y, z)=\frac{1}{2}\left[\exp \left(\frac{2 \pi \mathrm{i} y}{\varepsilon}\right)+1\right] K^{+}(y, z)+\left[\exp \left(\frac{2 \pi \mathrm{i} y}{\varepsilon}\right)-1\right] K^{-}(y, z) .
$$

\section{$7 \quad$ The transition layer $y=Y^{-}(z)$}

As we noted before, the functions $L^{ \pm}(y, z)$ are infinite on the curves $y=Y^{ \pm}(z)$. Hence, we need to find transition layer solutions there.

\subsection{The lower part $0<z<p$ (Region VIII)}

We introduce the stretched variable $\beta$, defined by

$$
y=Y^{-}(z)-\beta \varepsilon^{2 / 3} \quad \beta=O(1), \quad 0<z<p .
$$

Using (78) in (25)-(26) and expanding in powers of $\varepsilon$, with $\beta>0$, we get

$$
\psi^{-}(y, z) \sim \psi_{0}(z)+\ln \left(\frac{U_{0}+p}{U_{0}-q}\right) \beta \varepsilon^{2 / 3}-\frac{2}{3} \sqrt{\frac{U_{0}}{z}} \frac{1}{\left(U_{0}+p\right)\left(U_{0}-q\right)} \beta^{3 / 2} \varepsilon
$$

where

$$
\psi_{0}(z)=z \pi \mathrm{i}+(z-1) \ln \left(U_{0}\right)+Y^{-}(z) \ln \left(U_{0}-q\right)+\left[1-Y^{-}(z)\right] \ln \left(U_{0}+p\right)
$$

and

$$
L^{-}(y, z) \sim \frac{1}{\sqrt{2}} z^{-1 / 4} \sqrt{\left(U_{0}+p\right)\left(U_{0}-q\right)}\left(U_{0}\right)^{-3 / 4} \beta^{-1 / 4} \varepsilon^{-1 / 6}
$$


Hence,

$$
\begin{gathered}
K^{-}(y, z) \sim \varepsilon^{1 / 3} \frac{1}{2 \sqrt{\pi}} z^{-1 / 4} \sqrt{\left(U_{0}+p\right)\left(U_{0}-q\right)}\left(U_{0}\right)^{-3 / 4} \beta^{-1 / 4} \\
\times \exp \left[\varepsilon^{-1} \psi_{0}(z)+\ln \left(\frac{U_{0}+p}{U_{0}-q}\right) \beta \varepsilon^{-1 / 3}-\frac{2}{3} \sqrt{\frac{U_{0}}{z}} \frac{1}{\left(U_{0}+p\right)\left(U_{0}-q\right)} \beta^{3 / 2}\right]
\end{gathered}
$$

for $y \uparrow Y^{-}(z)$. Thus, we introduce the function $G^{(8)}(\beta, z)$ and consider solutions of the form

$$
K_{n}(x)=\varepsilon^{\nu_{8}} \exp \left[\psi_{0}(z) \varepsilon^{-1}+\ln \left(\frac{U_{0}+p}{U_{0}-q}\right)\left(Y^{-}-\varepsilon x\right) \varepsilon^{-1}\right] G^{(8)}\left(\frac{Y^{-}-\varepsilon x}{\varepsilon^{2 / 3}}, \varepsilon n\right) .
$$

Using (83) in (41) and expanding in powers of $\varepsilon$ we obtain

$$
G_{\beta \beta}^{(8)}=-\frac{2 \beta}{\left[\left(Y^{-}\right)^{\prime}\right]^{2}\left[(p-q) z+Y^{-}-p\right]} G^{(8)}=\beta \Theta^{2} G^{(8)}
$$

where

$$
\Theta(z)=\sqrt{\frac{U_{0}}{z}} \frac{1}{\left(U_{0}+p\right)\left(U_{0}-q\right)} .
$$

Solving (84) we get

$$
G^{(8)}(\beta, z)=A^{(8)}(z) \operatorname{Ai}\left[\Theta^{2 / 3} \beta\right]+B^{(8)}(z) \operatorname{Bi}\left[\Theta^{2 / 3} \beta\right]
$$

where $A^{(8)}(z), B^{(8)}(z)$ are functions to be determined and $\mathrm{Ai}(\cdot), \mathrm{Bi}(\cdot)$ are the Airy functions. Using the formulas [1]

$$
\begin{aligned}
& \operatorname{Ai}(x) \sim \frac{1}{2 \sqrt{\pi}} x^{-1 / 4} \exp \left(-\frac{2}{3} x^{3 / 2}\right), \quad x \rightarrow \infty \\
& \operatorname{Bi}(x) \sim \frac{1}{\sqrt{\pi}} x^{-1 / 4} \exp \left(\frac{2}{3} x^{3 / 2}\right), \quad x \rightarrow \infty
\end{aligned}
$$

in (86)) and matching with (82) we conclude that $\nu_{8}=1 / 3, B^{(8)}(z)=0$ and

$$
A^{(8)}(z)=\left[\frac{\left(U_{0}+p\right)\left(U_{0}-q\right)}{z\left(U_{0}\right)^{2}}\right]^{1 / 3}=\frac{\Theta^{-1 / 3}}{\sqrt{z U_{0}}} .
$$

Therefore,

$$
K^{(8)}(\beta, z)=\varepsilon^{1 / 3} \exp \left[\varepsilon^{-1} \psi_{0}(z)+\ln \left(\frac{U_{0}+p}{U_{0}-q}\right) \beta \varepsilon^{-1 / 3}\right] \operatorname{Ai}\left[\Theta^{2 / 3} \beta\right] \frac{\Theta^{-1 / 3}}{\sqrt{z U_{0}}}
$$

for $y-Y^{-}(z)=O\left(\varepsilon^{2 / 3}\right)$. 


\subsection{The upper part $p<z<1$ (Region IX)}

Using (78) in (25) we have, as $y \uparrow Y^{-}(z)$

$$
\begin{aligned}
& \psi^{-}(y, z) \sim \psi_{0}(z)+\ln \left(\frac{U_{0}+p}{U_{0}-q}\right) \beta \varepsilon^{2 / 3}+\frac{2}{3} \vartheta \beta^{3 / 2} \varepsilon \\
& \psi^{+}(y, z) \sim \psi_{0}(z)+\ln \left(\frac{U_{0}+p}{U_{0}-q}\right) \beta \varepsilon^{2 / 3}-\frac{2}{3} \vartheta \beta^{3 / 2} \varepsilon
\end{aligned}
$$

and from (26) we have

$$
\begin{aligned}
L^{-}(y, z) & \sim \frac{1}{\sqrt{2}} \frac{1}{\sqrt{z U_{0} \vartheta}} \mathrm{i} \beta^{-1 / 4} \varepsilon^{-1 / 6} \\
L^{+}(y, z) & \sim \frac{1}{\sqrt{2}} \frac{1}{\sqrt{z U_{0} \vartheta}} \beta^{-1 / 4} \varepsilon^{-1 / 6}
\end{aligned}
$$

where

$$
\vartheta(z)=-\Theta(z)=\sqrt{\frac{U_{0}}{z}} \frac{1}{\left(U_{0}+p\right)\left(q-U_{0}\right)} .
$$

Using (90)-(93) in (77) we have

$$
\begin{aligned}
K^{(7)}(y, z) & \sim \frac{1}{2 \sqrt{\pi}} \frac{1}{\sqrt{z U_{0} \vartheta}} \beta^{-1 / 4} \varepsilon^{1 / 3} \exp \left[\psi_{0}(z) \varepsilon^{-1}+\ln \left(\frac{U_{0}+p}{U_{0}-q}\right) \beta \varepsilon^{-1 / 3}\right] \\
\times & {\left[\frac{1}{2} \lambda^{+}(\beta, z) \exp \left(-\frac{2}{3} \vartheta \beta^{3 / 2}\right)+\mathrm{i} \lambda^{-}(\beta, z) \exp \left(\frac{2}{3} \vartheta \beta^{3 / 2}\right)\right] }
\end{aligned}
$$

as $y \uparrow Y^{-}(z)$, where

$$
\lambda^{ \pm}(\beta, z)=\exp \left\{\frac{2 \pi \mathrm{i}\left[Y^{-}(z)-\beta \varepsilon^{2 / 3}\right]}{\varepsilon}\right\} \pm 1 .
$$

We consider the ansatz

$$
\begin{aligned}
K_{n}(x) & \sim K^{(9)}(\beta, z)=\varepsilon^{\nu_{9}} \exp \left[\varepsilon^{-1} \psi_{0}(z)+\ln \left(\frac{U_{0}+p}{U_{0}-q}\right) \beta \varepsilon^{-1 / 3}\right] \\
& \times\left[\lambda^{+}(\beta, z) A^{(9)}(z) \operatorname{Ai}\left(\vartheta^{2 / 3} \beta\right)+\lambda^{-}(\beta, z) B^{(9)}(z) \operatorname{Bi}\left(\vartheta^{2 / 3} \beta\right)\right]
\end{aligned}
$$

for $y \approx Y^{-}(z), \quad p<z<1$ and unknown functions $A^{(9)}(z), B^{(9)}(z)$. Using (87) in (97) we have, as $\beta \rightarrow \infty$

$$
\begin{gathered}
K^{(9)}(\beta, z) \sim \varepsilon^{\nu_{9}} \exp \left[\varepsilon^{-1} \psi_{0}(z)+\ln \left(\frac{U_{0}+p}{U_{0}-q}\right) \beta \varepsilon^{-1 / 3}\right] \\
\times\left[\lambda^{+}(\beta, z) A^{(9)}(z) \frac{1}{2 \sqrt{\pi}} \vartheta^{-1 / 6} \beta^{-1 / 4} \exp \left(-\frac{2}{3} \vartheta \beta^{3 / 2}\right)+\right. \\
\left.\lambda^{-}(\beta, z) B^{(9)}(z) \frac{1}{\sqrt{\pi}} \vartheta^{-1 / 6} \beta^{-1 / 4} \exp \left(\frac{2}{3} \vartheta \beta^{3 / 2}\right)\right] .
\end{gathered}
$$


Matching (98) and (95) we get

$$
\nu_{9}=\frac{1}{3}, \quad A^{(9)}(z)=\frac{1}{2} \frac{\vartheta^{-1 / 3}}{\sqrt{z U_{0}}}, \quad B^{(9)}(z)=\frac{1}{2} \mathrm{i} \frac{\vartheta^{-1 / 3}}{\sqrt{z U_{0}}} .
$$

Thus,

$$
\begin{aligned}
K^{(9)}(\beta, z) & =\varepsilon^{1 / 3} \exp \left[\varepsilon^{-1} \psi_{0}(z)+\ln \left(\frac{U_{0}+p}{U_{0}-q}\right) \beta \varepsilon^{-1 / 3}\right] \\
& \times \frac{1}{2} \frac{\vartheta^{-1 / 3}}{\sqrt{z U_{0}}}\left[\lambda^{+}(\beta, z) \operatorname{Ai}\left(\vartheta^{2 / 3} \beta\right)+\mathrm{i} \lambda^{-}(\beta, z) \operatorname{Bi}\left(\vartheta^{2 / 3} \beta\right)\right] .
\end{aligned}
$$

\section{The interior of E (Region X)}

We shall now find an asymptotic solution for $Y^{-}(z)<y<Y^{+}(z)$. We set $\beta=-\widetilde{\beta}, \widetilde{\beta}>0$ in (79)-(81) and obtain, for $0<z<p$

$$
\psi^{-}(y, z) \sim \psi_{0}(z)-\ln \left(\frac{U_{0}+p}{U_{0}-q}\right) \widetilde{\beta} \varepsilon^{2 / 3}+\frac{2}{3} \Theta \mathrm{i} \widetilde{\beta}^{3 / 2} \varepsilon
$$

and

$$
L^{-}(y, z) \sim \frac{1}{\sqrt{2}} \frac{1}{\sqrt{z U_{0} \Theta}} e^{-\frac{1}{4} \pi \mathrm{i}} \widetilde{\beta}^{-1 / 4} \varepsilon^{-1 / 6} .
$$

Similarly, from (25)-(26) we obtain, for $0<z<p$

$$
\psi^{+}(y, z) \sim \psi_{0}(z)-\ln \left(\frac{U_{0}+p}{U_{0}-q}\right) \widetilde{\beta} \varepsilon^{2 / 3}-\frac{2}{3} \Theta \mathrm{i} \widetilde{\beta}^{3 / 2} \varepsilon
$$

and

$$
L^{+}(y, z) \sim \frac{1}{\sqrt{2}} \frac{1}{\sqrt{z U_{0} \Theta}} e^{\frac{1}{4} \pi \mathrm{i}} \widetilde{\beta}^{-1 / 4} \varepsilon^{-1 / 6} .
$$

Introducing the function $K^{(10)}(y, z)$ defined by

$$
\begin{gathered}
K^{(10)}(y, z)=\varepsilon^{\nu_{10}} A^{(10)}(y) \exp \left[\varepsilon^{-1} \psi^{-}(y, z)\right] L^{-}(y, z) \\
+\varepsilon^{\nu_{10}} B^{(10)}(y) \exp \left[\varepsilon^{-1} \psi^{+}(y, z)\right] L^{+}(y, z)
\end{gathered}
$$

with $A^{(10)}(y), B^{(10)}(y)$ to be determined, we have

$$
\begin{aligned}
K^{(10)}(y, z) & \sim \varepsilon^{\nu_{10}-1 / 6} \frac{1}{\sqrt{2}} \frac{1}{\sqrt{z U_{0} \Theta}} \widetilde{\beta}^{-1 / 4} \exp \left[\psi_{0}^{-}(z) \varepsilon^{-1}-\ln \left(\frac{U_{0}+p}{U_{0}-q}\right) \widetilde{\beta} \varepsilon^{-1 / 3}\right] \\
& \times\left[A^{(10)}(y) \exp \left(\frac{2}{3} \Theta \mathrm{i} \widetilde{\beta}^{3 / 2}-\frac{1}{4} \pi \mathrm{i}\right)+B^{(10)}(y) \exp \left(-\frac{2}{3} \Theta \mathrm{i} \widetilde{\beta}^{3 / 2}+\frac{1}{4} \pi \mathrm{i}\right)\right] .
\end{aligned}
$$


From (89) we have

$$
\begin{aligned}
K^{(9)}(\beta, z) & \sim \varepsilon^{1 / 3} \frac{1}{\sqrt{\pi}} \frac{1}{\sqrt{z U_{0} \Theta}} \widetilde{\beta}^{-1 / 4} \exp \left[\varepsilon^{-1} \psi_{0}^{-}(z)-\ln \left(\frac{U_{0}+p}{U_{0}-q}\right) \widetilde{\beta} \varepsilon^{-1 / 3}\right] \\
& \times \sin \left(\frac{2}{3} \Theta \widetilde{\beta}^{3 / 2}+\frac{\pi}{4}\right)
\end{aligned}
$$

where we have used the asymptotic formula [1]

$$
\operatorname{Ai}(-x) \sim \frac{x^{-1 / 4}}{\sqrt{\pi}} \sin \left(\frac{2}{3} x^{3 / 2}+\frac{\pi}{4}\right), \quad x \rightarrow \infty .
$$

Matching (105) and (106) we get $\nu_{10}=\frac{1}{2}$ and

$$
\begin{aligned}
& \frac{1}{\sqrt{2}}\left[A^{(10)}(y) \exp \left(\frac{2}{3} \Theta \mathrm{i} \widetilde{\beta}^{3 / 2}-\frac{1}{4} \pi \mathrm{i}\right)+B^{(10)}(y) \exp \left(-\frac{2}{3} \Theta \mathrm{i} \widetilde{\beta}^{3 / 2}+\frac{1}{4} \pi \mathrm{i}\right)\right] \\
& =\frac{1}{\sqrt{\pi}} \sin \left(\frac{2}{3} \Theta \widetilde{\beta}^{3 / 2}+\frac{\pi}{4}\right)=\frac{1}{2 \sqrt{\pi}}\left[\exp \left(\frac{2}{3} \Theta \mathrm{i} \widetilde{\beta}^{3 / 2}-\frac{1}{4} \pi \mathrm{i}\right)+\exp \left(-\frac{2}{3} \Theta \mathrm{i} \widetilde{\beta}^{3 / 2}+\frac{1}{4} \pi \mathrm{i}\right)\right]
\end{aligned}
$$

from which we conclude that

$$
A^{(10)}(y)=\frac{1}{\sqrt{2 \pi}}=B^{(10)}(y)
$$

and therefore

$$
K_{n}(x) \sim K^{(10)}(y, z)=K^{+}(y, z)+K^{-}(y, z)
$$

for $Y^{-}(z)<y<Y^{+}(z)$.

\subsection{Matching the interior of $\mathrm{E}$ and the upper part of the transition layer $Y^{-}$}

We shall now verify the matching between (108) and (99). For $p<z<1$ we have

$$
\begin{gathered}
\psi^{-}(y, z) \sim\left(Y^{-}+\widetilde{\beta} \varepsilon^{2 / 3}-z\right) 2 \pi \mathrm{i}+\psi_{0}(z)-\ln \left(\frac{U_{0}+p}{U_{0}-q}\right) \widetilde{\beta} \varepsilon^{2 / 3}-\frac{2}{3} \vartheta \mathrm{i} \widetilde{\beta}^{3 / 2} \varepsilon, \\
L^{-}(y, z) \sim \frac{1}{\sqrt{2}} \frac{1}{\sqrt{z U_{0} \vartheta}} e^{\frac{1}{4} \pi \mathrm{i}} \widetilde{\beta}^{-1 / 4} \varepsilon^{-1 / 6} \\
\psi^{+}(y, z) \sim \psi_{0}(z)-\ln \left(\frac{U_{0}+p}{U_{0}-q}\right) \widetilde{\beta} \varepsilon^{2 / 3}+\frac{2}{3} \vartheta \mathrm{i} \widetilde{\beta}^{3 / 2} \varepsilon
\end{gathered}
$$

and

$$
L^{+}(y, z) \sim \frac{1}{\sqrt{2}} \frac{1}{\sqrt{z U_{0} \vartheta}} e^{-\frac{1}{4} \pi \mathrm{i}} \widetilde{\beta}^{-1 / 4} \varepsilon^{-1 / 6} .
$$


Therefore,

$$
\begin{gathered}
K^{(10)}(y, z) \sim \frac{\varepsilon^{1 / 3}}{2 \sqrt{\pi}} \frac{\widetilde{\beta}^{-1 / 4}}{\sqrt{z U_{0} \vartheta}} \exp \left[\varepsilon^{-1} \psi_{0}(z)-\ln \left(\frac{U_{0}+p}{U_{0}-q}\right) \widetilde{\beta} \varepsilon^{-1 / 3}\right] \\
\times\left[\frac{\lambda^{+}(-\widetilde{\beta}, z)+\lambda^{-}(-\widetilde{\beta}, z)}{2} \exp \left(-\frac{2}{3} \vartheta \mathrm{i} \widetilde{\beta}^{3 / 2}+\frac{1}{4} \pi \mathrm{i}\right)+\exp \left(\frac{2}{3} \vartheta \mathrm{i} \widetilde{\beta}^{3 / 2}-\frac{1}{4} \pi \mathrm{i}\right)\right] .
\end{gathered}
$$

Using (107) and [1]

$$
\operatorname{Bi}(-x) \sim \frac{x^{-1 / 4}}{\sqrt{\pi}} \cos \left(\frac{2}{3} x^{3 / 2}+\frac{\pi}{4}\right), \quad x \rightarrow \infty
$$

in (99) we have

$$
\begin{gathered}
K^{(9)}(\beta, z) \sim \varepsilon^{1 / 3} \exp \left[\varepsilon^{-1} \psi_{0}(z)-\ln \left(\frac{U_{0}+p}{U_{0}-q}\right) \widetilde{\beta} \varepsilon^{-1 / 3}\right] \\
\times \frac{1}{2 \sqrt{\pi}} \frac{\widetilde{\beta}^{-1 / 4}}{\sqrt{z U_{0} \vartheta}}\left[\lambda^{+}(-\widetilde{\beta}, z) \sin \left(\frac{2}{3} \vartheta \widetilde{\beta}^{3 / 2}+\frac{\pi}{4}\right)+\mathrm{i} \lambda^{-}(-\widetilde{\beta}, z) \cos \left(\frac{2}{3} \vartheta \widetilde{\beta}^{3 / 2}+\frac{\pi}{4}\right)\right]
\end{gathered}
$$

or

$$
\begin{aligned}
& K^{(9)}(\beta, z) \sim \varepsilon^{1 / 3} \exp \left[\varepsilon^{-1} \psi_{0}(z)-\ln \left(\frac{U_{0}+p}{U_{0}-q}\right) \widetilde{\beta} \varepsilon^{-1 / 3}\right] \frac{1}{2 \sqrt{\pi}} \frac{\widetilde{\beta}^{-1 / 4}}{\sqrt{z U_{0} \vartheta}} \\
\times & {\left[\frac{\lambda^{+}+\lambda^{-}}{2} \exp \left(-\frac{2}{3} \vartheta \mathrm{i} \widetilde{\beta}^{3 / 2}+\frac{1}{4} \pi \mathrm{i}\right)+\frac{\lambda^{+}-\lambda^{-}}{2} \exp \left(\frac{2}{3} \vartheta \mathrm{i} \widetilde{\beta}^{3 / 2}-\frac{1}{4} \pi \mathrm{i}\right)\right] . }
\end{aligned}
$$

Since from (96) we have

$$
\frac{\lambda^{+}-\lambda^{-}}{2}=1
$$

we see that (114) agrees with (113).

\subsection{Matching the interior of $\mathbf{E}$ and the corner layer at $(p, 0)$}

Using (33) and the asymptotic formula [19]

$$
H_{n}(x) \sim \sqrt{2}^{n+1} n^{n / 2} \exp \left(\frac{x^{2}}{2}-\frac{n}{2}\right) \cos \left(\sqrt{2 n} x-\frac{n \pi}{2}\right), \quad n \rightarrow \infty
$$

in (31) we have, as $n \rightarrow \infty$

$$
K_{n}^{(2)}(\eta) \sim \frac{1}{\sqrt{n \pi}} \exp \left\{\frac{\eta^{2}}{2}+\frac{n}{2}\left[1+\ln \left(\frac{p q}{\varepsilon n}\right)\right]\right\} \cos \left(\sqrt{2 n} \eta-\frac{n \pi}{2}\right) .
$$


Using (30) in (25), with $z=n \varepsilon$ we get

$$
\frac{1}{\varepsilon} \psi^{ \pm} \sim \frac{n}{2}\left[1+\ln \left(\frac{p q}{\varepsilon n}\right) \pm \pi \mathrm{i}\right] \mp \sqrt{2 n} \eta \mathrm{i}+\frac{\eta^{2}}{2} .
$$

Similarly from (26) we find

$$
L^{+} \sim \frac{1}{\sqrt{2 n}}, \quad L^{-} \sim \frac{1}{\sqrt{2 n}}
$$

Using (116)-(117) in (108) we obtain

$$
K^{(10)} \sim \frac{1}{\sqrt{\pi n}} \exp \left\{\frac{\eta^{2}}{2}+\frac{n}{2}\left[1+\ln \left(\frac{p q}{\varepsilon n}\right)\right]\right\} \cos \left(\sqrt{2 n} \eta-\frac{n \pi}{2}\right)
$$

which is in agreement with (115).

\section{The boundary layer at $z=1$ (Region XI)}

We now consider solutions of (4) with $n \approx N$. We introduce the variable $j$ and the function $\Upsilon_{j}^{(11)}(y)$ defined by

$$
\begin{gathered}
n=N-j, \quad j \in \mathbb{Z}, \quad j \geq-1, \\
K_{n}(x)=\left(\begin{array}{c}
N \\
N-n
\end{array}\right)(-p)^{n} \Upsilon_{N-n}^{(11)}\left(\frac{x}{N}\right) .
\end{gathered}
$$

Using (119) in (4) we have, to leading order,

$$
q \Upsilon_{j+1}^{(11)}-(q-y) \Upsilon_{j}^{(11)}=0
$$

which can be solved to obtain

$$
\Upsilon_{j}^{(11)}(y)=A^{(11)}(y)\left(1-\frac{y}{q}\right)^{j}
$$

where $A^{(11)}(y)$ is a function to be determined. Thus, we have

$$
K_{n}(x) \sim A^{(11)}(y)\left(\begin{array}{c}
N \\
j
\end{array}\right)(-p)^{N-j}\left(1-\frac{y}{q}\right)^{j} .
$$

We now introduce the function $R_{j}^{(11)}(y)$ defined by

$$
K_{n}(x)=\left(\begin{array}{l}
x \\
n
\end{array}\right) R_{N-n}^{(11)}\left(\frac{x}{N}\right)
$$

and note that from (??) we have

$$
R_{-1}^{(11)}(y)=1
$$


Using (118) in (4) we obtain, to leading order,

$$
(q-y) R_{j+1}^{(11)}-(1-y) R_{j}^{(11)}=0
$$

which together with (123) implies

$$
R_{j}^{(11)}(y)=\left(\frac{1-y}{q-y}\right)^{j+1}, \quad y \neq q
$$

Therefore,

$$
K_{n}(x) \sim\left(\begin{array}{c}
N y \\
n
\end{array}\right)\left(\frac{1-y}{q-y}\right)^{j+1}, \quad y \not \approx q .
$$

From (122) and (125) we get, for $y \not \approx q$,

$$
K_{n}(x) \sim K_{j}^{(11)}(y)=A^{(11)}(y)\left(\begin{array}{c}
N \\
j
\end{array}\right)(-p)^{N-j}\left(1-\frac{y}{q}\right)^{j}+\left(\begin{array}{c}
N y \\
N-j
\end{array}\right)\left(\frac{1-y}{q-y}\right)^{j+1} .
$$

\subsection{Matching with the upper corners}

We shall now determine the function $A^{(11)}(y)$ by matching (126) with (176). As $N \rightarrow \infty$ we get

$$
\begin{aligned}
& K_{j}^{(11)}(y) \sim A^{(11)}(y) \frac{1}{\sqrt{2 \pi j}} \exp \left\{\varepsilon^{-1} \ln (-p)+\left[1-\ln (j \varepsilon)-\ln (-p)+\ln \left(1-\frac{y}{q}\right)\right] j\right\} \\
& +\varepsilon^{1 / 2} \sqrt{\frac{2}{\pi}} \frac{\sqrt{(1-y) y}}{y-q} \sin (N \pi y) \exp \left\{\frac{y \ln (y)+(1-y) \ln (1-y)+\pi \mathrm{i}}{\varepsilon}-j \ln (y-q)\right\} .
\end{aligned}
$$

Using (118) in (25) we have as $\varepsilon \rightarrow 0,0<y<q$

$$
\begin{gathered}
\psi^{+}(y, z) \sim \ln (-p)+y \ln \left(\frac{q}{p}\right)-y \pi \mathrm{i}+\left[1-\ln (j \varepsilon)-\ln (-p)+\ln \left(1-\frac{y}{q}\right)\right] j \varepsilon \\
\psi^{-}(y, z) \sim y \ln (y)+(1-y) \ln (1-y)+(1-y) \pi \mathrm{i}-j \ln (y-q) \varepsilon
\end{gathered}
$$

and from (26) we get

$$
L^{+}(y, z) \sim \frac{1}{\sqrt{j \varepsilon}}, \quad L^{-}(y, z) \sim \sqrt{y(1-y)} \frac{1}{q-y} \mathrm{i} .
$$

Using (128)-(130) in (76) we have

$$
\begin{aligned}
& K^{(7)}(y, z) \sim \cos \left(\frac{\pi y}{\varepsilon}\right) \frac{1}{\sqrt{2 \pi j}} \exp \left\{\frac{\ln (-p)+y \ln \left(\frac{q}{p}\right)}{\varepsilon}+\left[1-\ln (j \varepsilon)-\ln (-p)+\ln \left(1-\frac{y}{q}\right)\right] j\right\} \\
& +\sqrt{y(1-y)} \frac{1}{y-q} \sin \left(\frac{\pi y}{\varepsilon}\right) \sqrt{\frac{2 \varepsilon}{\pi}} \exp \left[\frac{y \ln (y)+(1-y) \ln (1-y)+\pi \mathrm{i}}{\varepsilon}-j \ln (y-q)\right]
\end{aligned}
$$


Matching (127) and 131 we obtain

$$
A^{(11)}(y)=\exp \left[y \ln \left(\frac{q}{p}\right) \varepsilon^{-1}\right] \cos \left(\frac{\pi y}{\varepsilon}\right) .
$$

Hence, we conclude that

$$
K_{j}^{(11)}(y)=\left(\begin{array}{c}
N \\
j
\end{array}\right)(-p)^{N-j}\left(\frac{q}{p}\right)^{N y} \cos (N \pi y)\left(1-\frac{y}{q}\right)^{j}+\left(\begin{array}{c}
N y \\
N-j
\end{array}\right)\left(\frac{1-y}{q-y}\right)^{j+1}
$$

for $z=1-O(\varepsilon)$ and $y \not \approx q$.

\subsection{The corner layer at $(q, 1)$ (Region XII)}

The approximation (133) ceases to be valid for $y \approx q$. Therefore, we need to find another expression, which holds in a neighborhood of the point $(q, 1)$. We introduce the new variable $\xi$ defined by

$$
y=q+\xi \sqrt{2 p q \varepsilon}, \quad \xi=O(1) .
$$

Using (134) in (133) we have, as $\varepsilon \rightarrow 0$

$$
\begin{gathered}
K_{j}^{(11)}(y) \sim \exp \left[\frac{p \ln (p)+q \ln (q)}{\varepsilon}+\xi \sqrt{\frac{2 p q}{\varepsilon}} \ln \left(\frac{q}{p}\right)-\frac{j}{2} \ln (p q \varepsilon)\right] \\
\times\left[\frac{(\sqrt{2} \xi)^{j}}{j !} \cos \left(\frac{p \pi}{\varepsilon}-\xi \pi \sqrt{\frac{2 p q}{\varepsilon}}\right)-\sqrt{\frac{2}{\pi}} e^{\xi^{2}}(\sqrt{2} \xi)^{-j-1} \sin \left(\frac{p \pi}{\varepsilon}-\xi \pi \sqrt{\frac{2 p q}{\varepsilon}}\right)\right] .
\end{gathered}
$$

Equation (135) suggests that we define a new function $R_{j}^{(12)}(\xi)$ by

$$
K_{n}(x)=\exp \left[\frac{p \ln (p)+q \ln (q)}{\varepsilon}+\xi \sqrt{\frac{2 p q}{\varepsilon}} \ln \left(\frac{q}{p}\right)-\frac{j}{2} \ln (p q \varepsilon)\right] R_{j}^{(12)}(\xi) .
$$

Using (136) in (44) we obtain, to leading order,

$$
(j+1) R_{j+1}^{(12)}-\sqrt{2} \xi R_{j}+R_{j-1}^{(12)}=0
$$

which has the independent solutions [38]

$$
R_{j}^{(12)}(\xi)=\frac{1}{j !} \mathrm{D}_{j}(\sqrt{2} \xi) \text {, and } R_{j}^{(12)}(\xi)=\mathrm{D}_{-j-1}( \pm \sqrt{2} \mathrm{i} \xi)( \pm \mathrm{i})^{j} .
$$

Using (65) and matching with (135) we get

$$
\begin{aligned}
K_{n}(x) & \sim K_{j}^{(12)}(\xi)=\exp \left[\frac{p \ln (p)+q \ln (q)}{\varepsilon}+\xi \sqrt{\frac{2 p q}{\varepsilon}} \ln \left(\frac{q}{p}\right)-\frac{j}{2} \ln (p q \varepsilon)\right] \exp \left(\frac{\xi^{2}}{2}\right) \\
& \times\left[\frac{1}{j !} \mathrm{D}_{j}(\sqrt{2} \xi) \cos \left(\frac{p \pi}{\varepsilon}-\xi \pi \sqrt{\frac{2 p q}{\varepsilon}}\right)-\frac{1}{\sqrt{2 \pi}} \Lambda_{j}(\xi) \sin \left(\frac{p \pi}{\varepsilon}-\xi \pi \sqrt{\frac{2 p q}{\varepsilon}}\right)\right],
\end{aligned}
$$


where the function $\Lambda_{j}: \mathbb{R} \rightarrow \mathbb{R}$ is defined by

$$
\Lambda_{j}(\xi)=\mathrm{i}^{j+1}\left[\mathrm{D}_{-j-1}(\sqrt{2} \mathrm{i} \xi)+(-1)^{j+1} \mathrm{D}_{-j-1}(-\sqrt{2} \mathrm{i} \xi)\right] .
$$

\subsubsection{Matching the corner at $(q, 1)$ and the interior of $\mathbf{E}$}

Finally, we verify the matching between (108) and (137). Using (118) and (134) in (25) we have,

$$
\begin{gathered}
\frac{\psi^{+}}{\varepsilon} \sim \frac{q \ln (q)+p \ln (p)+p \pi \mathrm{i}}{\varepsilon}+\xi \sqrt{2 p q}\left[\ln \left(\frac{q}{p}\right)-\pi \mathrm{i}\right] \varepsilon^{-1 / 2} \\
+\frac{j}{2}[1-\ln (p q j \varepsilon)-\pi \mathrm{i}]+\sqrt{2 j} \xi \mathrm{i}+\frac{\xi^{2}}{2} \\
\frac{\psi^{-}}{\varepsilon} \sim \frac{q \ln (q)+p \ln (p)-p \pi \mathrm{i}}{\varepsilon}+\xi \sqrt{2 p q}\left[\ln \left(\frac{q}{p}\right)+\pi \mathrm{i}\right] \varepsilon^{-1 / 2} \\
+\frac{j}{2}[1-\ln (p q j \varepsilon)+\pi \mathrm{i}]-\sqrt{2 j} \xi \mathrm{i}+\frac{\xi^{2}}{2} .
\end{gathered}
$$

Similarly, from (26) we get

and therefore

$$
L^{+} \sim \frac{1}{\sqrt{2 j \varepsilon}}, \quad L^{-} \sim \frac{1}{\sqrt{2 j \varepsilon}}
$$

$$
\begin{aligned}
& K^{(10)}(y, z) \sim \frac{1}{\sqrt{\pi j}} \exp \left[\frac{q \ln (q)+p \ln (p)}{\varepsilon}+\xi \sqrt{2 p q} \ln \left(\frac{q}{p}\right) \varepsilon^{-1 / 2}+\frac{\xi^{2}}{2}\right] \\
& \times \exp \left\{\frac{j}{2}[1-\ln (p q j \varepsilon)]\right\} \cos \left[\frac{p \pi}{\varepsilon}-\xi \sqrt{2 p q} \pi \varepsilon^{-1 / 2}+\sqrt{2 j} \xi-\frac{j \pi}{2}\right] .
\end{aligned}
$$

Using the formula [38]

$$
\mathrm{D}_{n}(\xi) \sim \sqrt{2} \exp \left\{\frac{n}{2}[\ln (n)-1]\right\} \cos \left[\sqrt{n} \xi-\frac{n \pi}{2}\right], \quad n \rightarrow \infty
$$

in (137) yields

$$
\begin{gathered}
K_{j}^{(12)}(\xi) \sim \exp \left[\frac{p \ln (p)+q \ln (q)}{\varepsilon}+\xi \sqrt{\frac{2 p q}{\varepsilon}} \ln \left(\frac{q}{p}\right)-\frac{j}{2} \ln (p q \varepsilon)\right] \frac{1}{\sqrt{\pi j}} \exp \left\{\frac{\xi^{2}}{2}+\frac{j}{2}[1-\ln (j)]\right\} \\
\times\left[\cos \left(\sqrt{j} \xi-\frac{j \pi}{2}\right) \cos \left(\frac{p \pi}{\varepsilon}-\xi \pi \sqrt{\frac{2 p q}{\varepsilon}}\right)-\sin \left(\sqrt{j} \xi-\frac{j \pi}{2}\right) \sin \left(\frac{p \pi}{\varepsilon}-\xi \pi \sqrt{\frac{2 p q}{\varepsilon}}\right)\right],
\end{gathered}
$$

where we have used

$$
\Lambda_{j}(\xi) \sim \sqrt{\frac{2}{j}} \exp \left\{\frac{j}{2}[1-\ln (j)]\right\} \sin \left(\sqrt{j} \xi-\frac{j \pi}{2}\right) .
$$

Equations (139) and (140) are identical, after regrouping terms. 


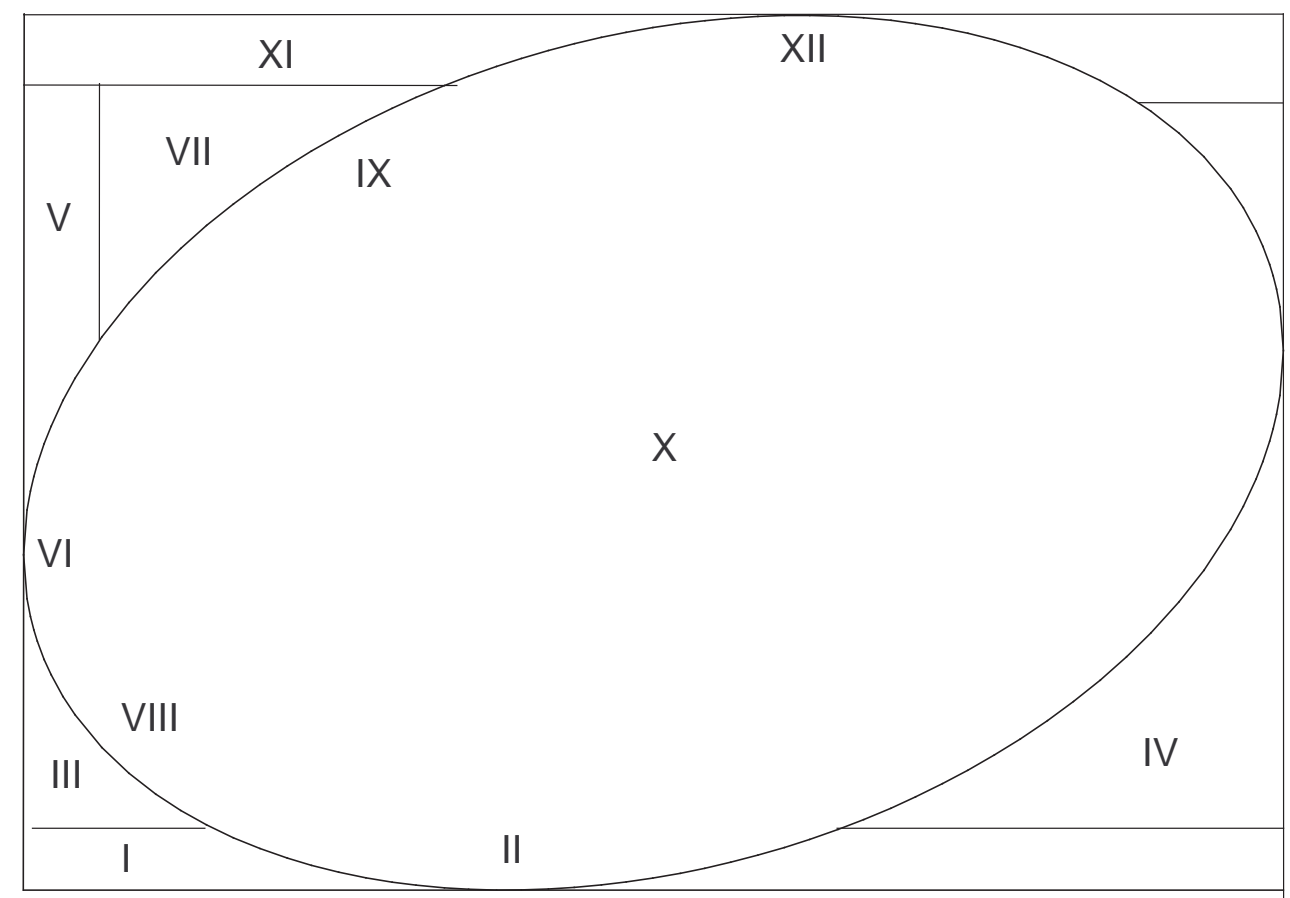

Figure 2: A sketch of the different asymptotic regions.

\section{Summary and numerical results}

Below we summarize our results for the various asymptotic approximations to $K_{n}(x)$ as $N \rightarrow \infty$, with

$$
\varepsilon=\frac{1}{N}, \quad x=\frac{y}{\varepsilon}, \quad n=\frac{z}{\varepsilon} \quad 0 \leq y, z \leq 1
$$

(see Figure 2).

1. Region I: $n=O(1), 0 \leq y \leq 1, y \not \approx p$.

$$
K_{n}(x) \sim K_{n}^{(1)}(y)=\frac{\varepsilon^{-n}}{n !}(y-p)^{n} .
$$

(see Figure 3). 


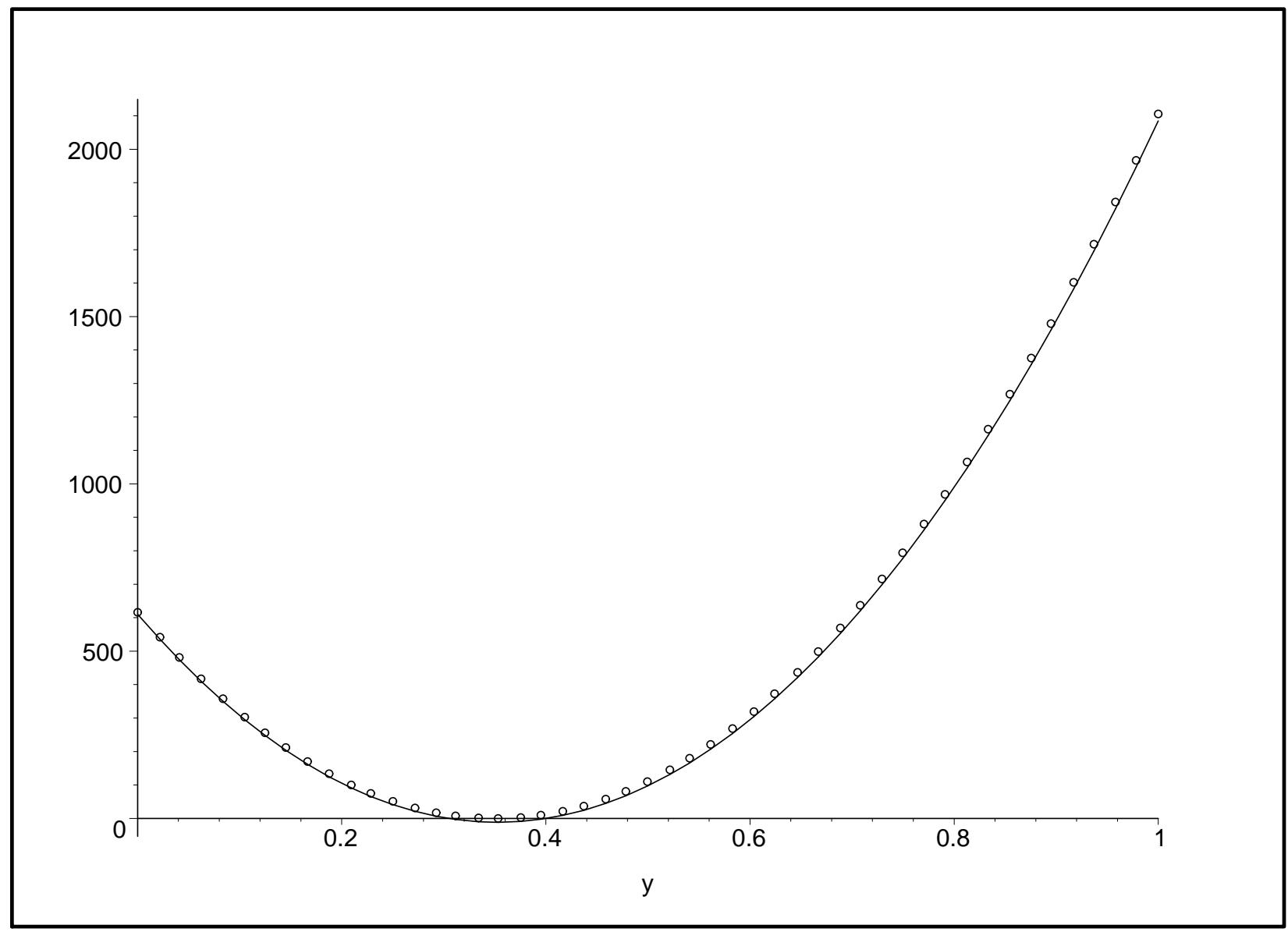

Figure 3: A comparison of $K_{n}(x)$ (solid curve) and $K_{n}^{(1)}(y)$ (ooo) for $n=2$ with $\varepsilon=0.01$ and $q=0.64894783$. 


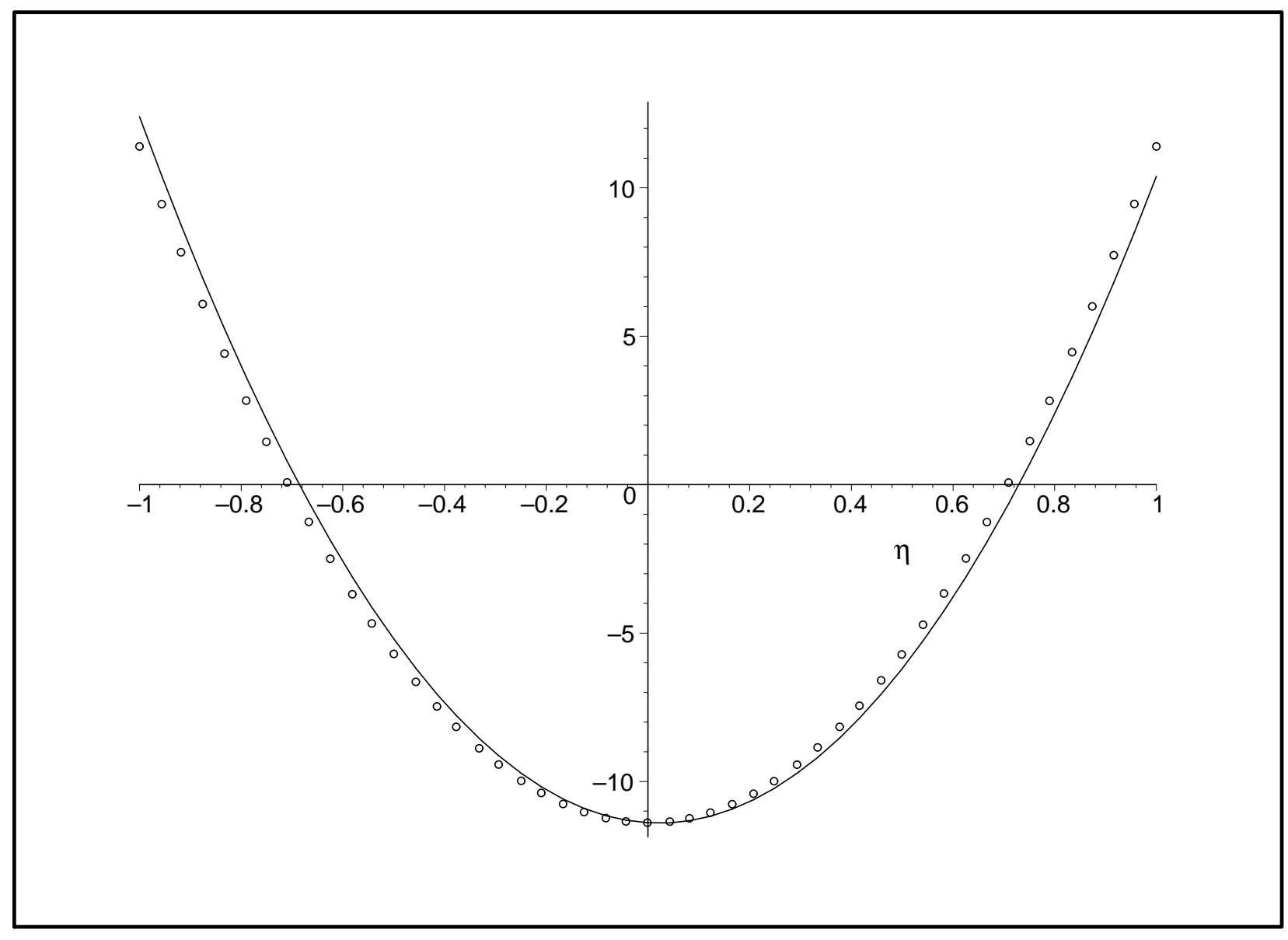

Figure 4: A comparison of $K_{n}(x)$ (solid curve) and $K_{n}^{(2)}(\eta)$ (ooo) for $n=2$ with $\varepsilon=0.01$ and $q=0.64894783$.

2. Region II: $n=O(1), y \approx p, y=p+\eta \sqrt{2 p q \varepsilon}, \eta=O(1)$.

$$
K_{n}(x) \sim K_{n}^{(2)}(\eta)=\frac{\varepsilon^{-n / 2}}{n !}\left(\frac{p q}{2}\right)^{n / 2} H_{n}(\eta)
$$

where $H_{n}(\eta)$ is the Hermite polynomial (see Figure 4).

3. Region III: $0 \leq y<Y^{-}(z), 0<z<p$, where

$$
\begin{gathered}
Y^{ \pm}(z)=p+(q-p) z \pm 2 z U_{0}, \quad U_{0}(z)=\sqrt{\frac{p q(1-z)}{z}} . \\
K_{n}(x) \sim K^{-}(y, z)=\varepsilon^{1 / 2} \frac{1}{\sqrt{2 \pi}} \exp \left[\varepsilon^{-1} \psi^{-}(y, z)\right] L^{-}(y, z),
\end{gathered}
$$




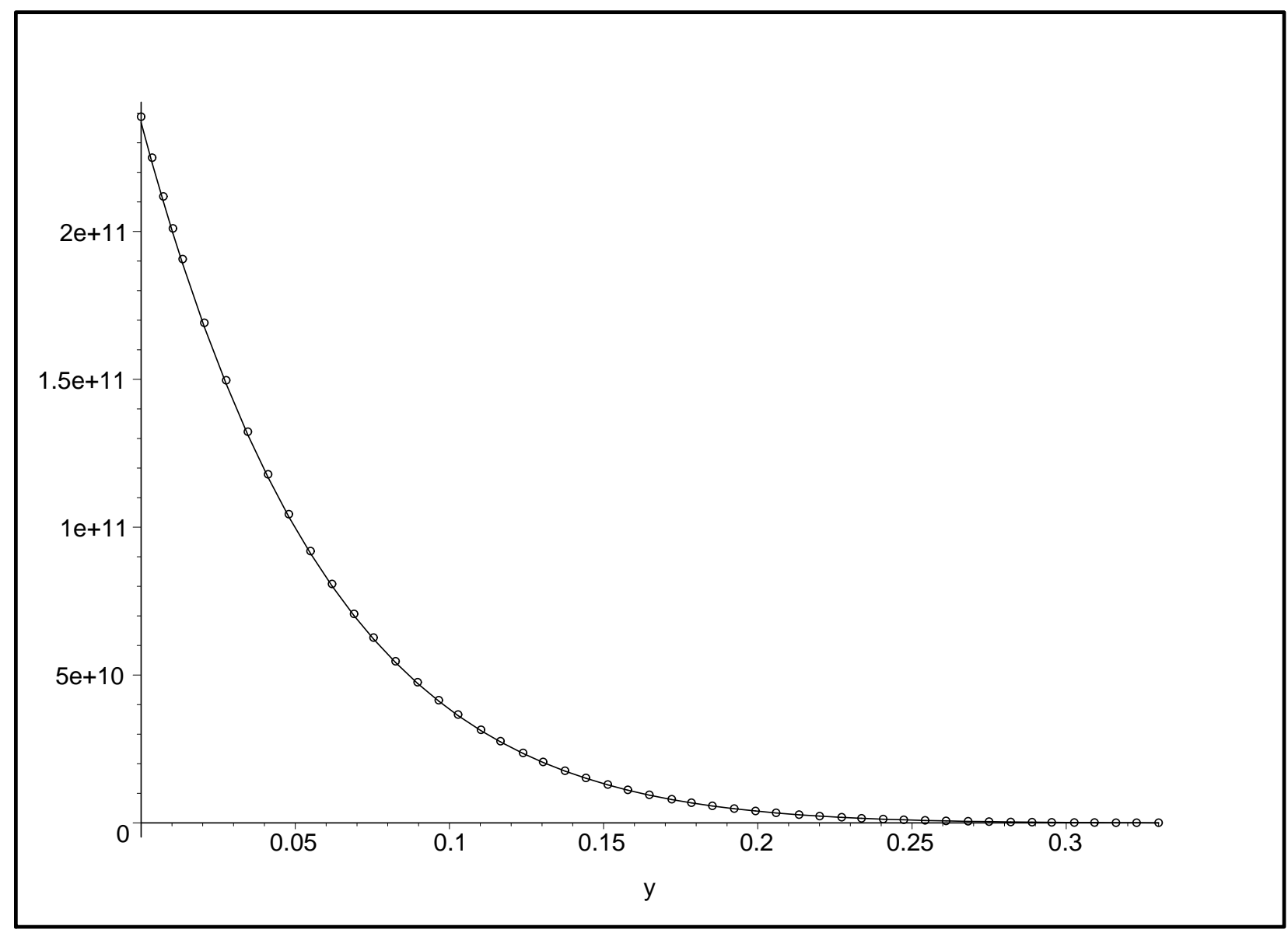

Figure 5: A comparison of $K_{n}(x)$ (solid curve) and $K^{-}(y, z)$ (ooo) for $n=10$ with $\varepsilon=0.01$ and $q=0.34894783$.

with

$$
\psi^{ \pm}(y, z)=\ln \left[\left(U^{ \pm}\right)^{z-1}\left(U^{ \pm}-p\right)^{1-y}\left(U^{ \pm}+q\right)^{y}\right], \quad L^{ \pm}(y, z)=\sqrt{\frac{\left(U^{ \pm}-p\right)\left(U^{ \pm}+q\right)}{z\left[\left(U^{ \pm}\right)^{2}-U_{0}^{2}\right]}}
$$

and

$$
U^{ \pm}(y, z)=-\frac{1}{2}\left(\frac{p-y}{z}+q-p\right) \pm \frac{1}{2} \sqrt{\left(\frac{p-y}{z}+q-p\right)^{2}-4\left(U_{0}\right)^{2}}
$$

(see Figure 51).

4. Region IV: $Y^{+}(z)<y \leq 1,0<z<q$.

$$
K_{n}(x) \sim K^{+}(y, z)=\varepsilon^{1 / 2} \frac{1}{\sqrt{2 \pi}} \exp \left[\varepsilon^{-1} \psi^{+}(y, z)\right] L^{+}(y, z) .
$$




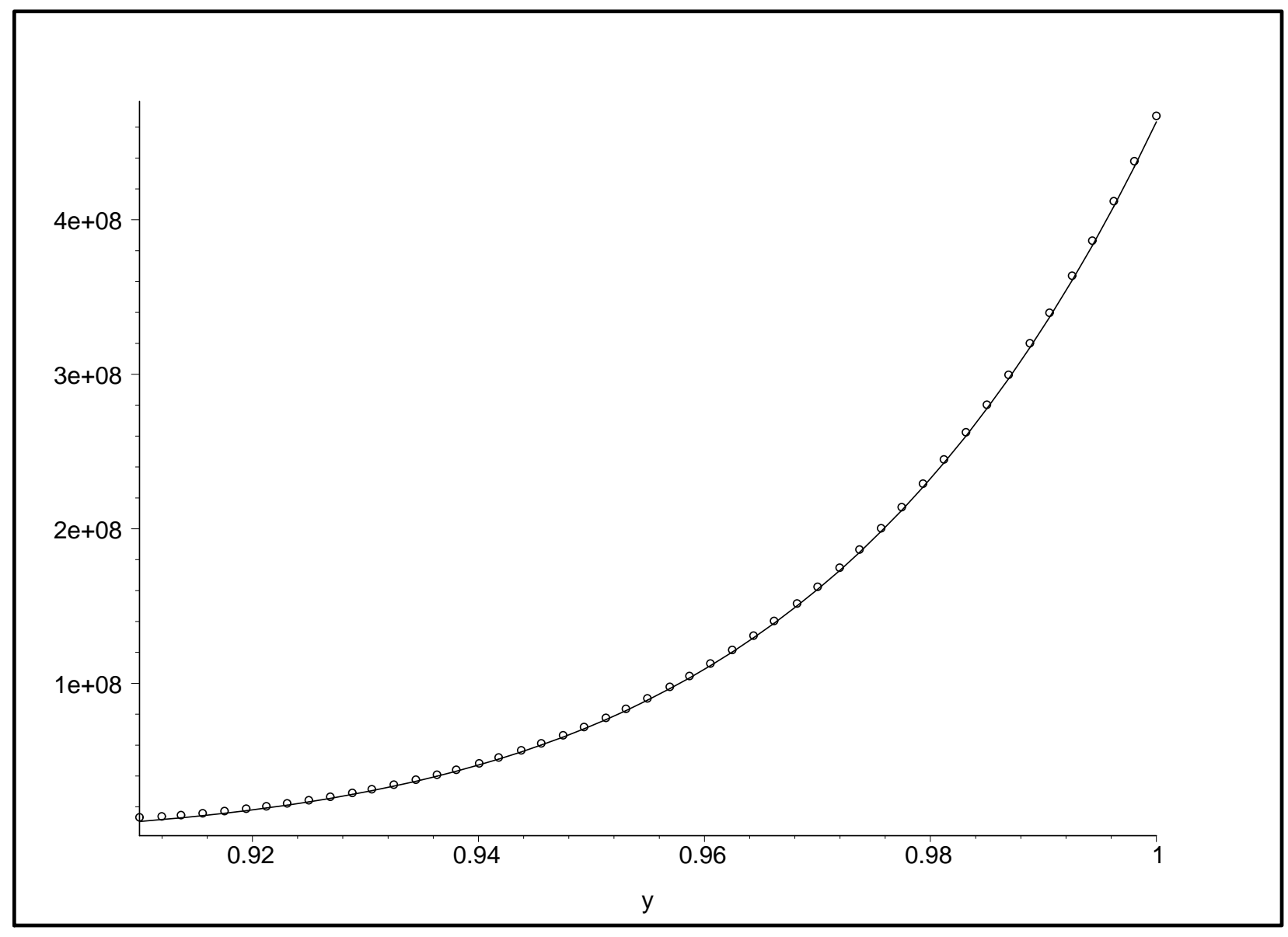

Figure 6: A comparison of $K_{n}(x)$ (solid curve) and $K^{+}(y, z)$ (ooo) for $n=10$ with $\varepsilon=0.01$ and $q=0.34894783$.

(see Figure 6).

5. Region V: $x=O(1), p<z<1$.

$$
\begin{aligned}
K_{n}(x) & \sim K^{(5)}(x, z)=\frac{\varepsilon^{1 / 2}}{\sqrt{2 \pi} \sqrt{z(1-z)}} \cos (\pi x)\left(\frac{z-p}{p}\right)^{x} \exp \left[\frac{\phi_{0}(z)}{\varepsilon}\right] \\
& -\frac{\varepsilon}{\pi} \frac{x}{z-p} \Gamma(x) \sin (\pi x)\left(\frac{q \varepsilon}{z-p}\right)^{x} \exp \left[\frac{(z-1) \ln (q)+\pi \mathrm{i} z}{\varepsilon}\right]
\end{aligned}
$$

where

$$
\phi_{0}(z)=(z-1) \ln (1-z)-z \ln (z)+z \ln (-p)
$$

and $\Gamma(x)$ is the Gamma function (see Figure 7 ). 


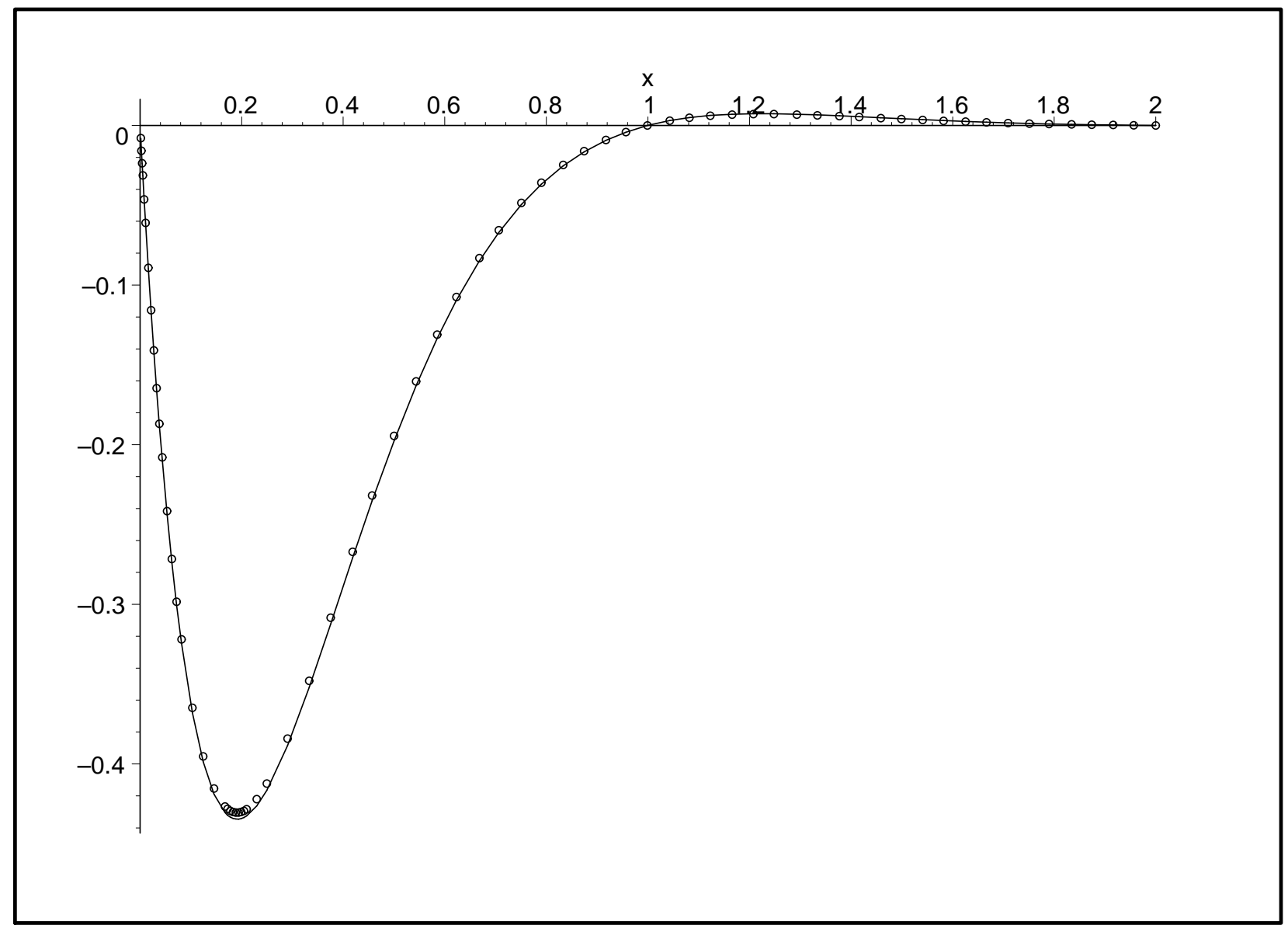

Figure 7: A comparison of $K_{n}(x)$ (solid curve) and $K^{(5)}(x, z)$ (ooo) for $n=80$ with $\varepsilon=0.01$ and $q=0.74894783$. 


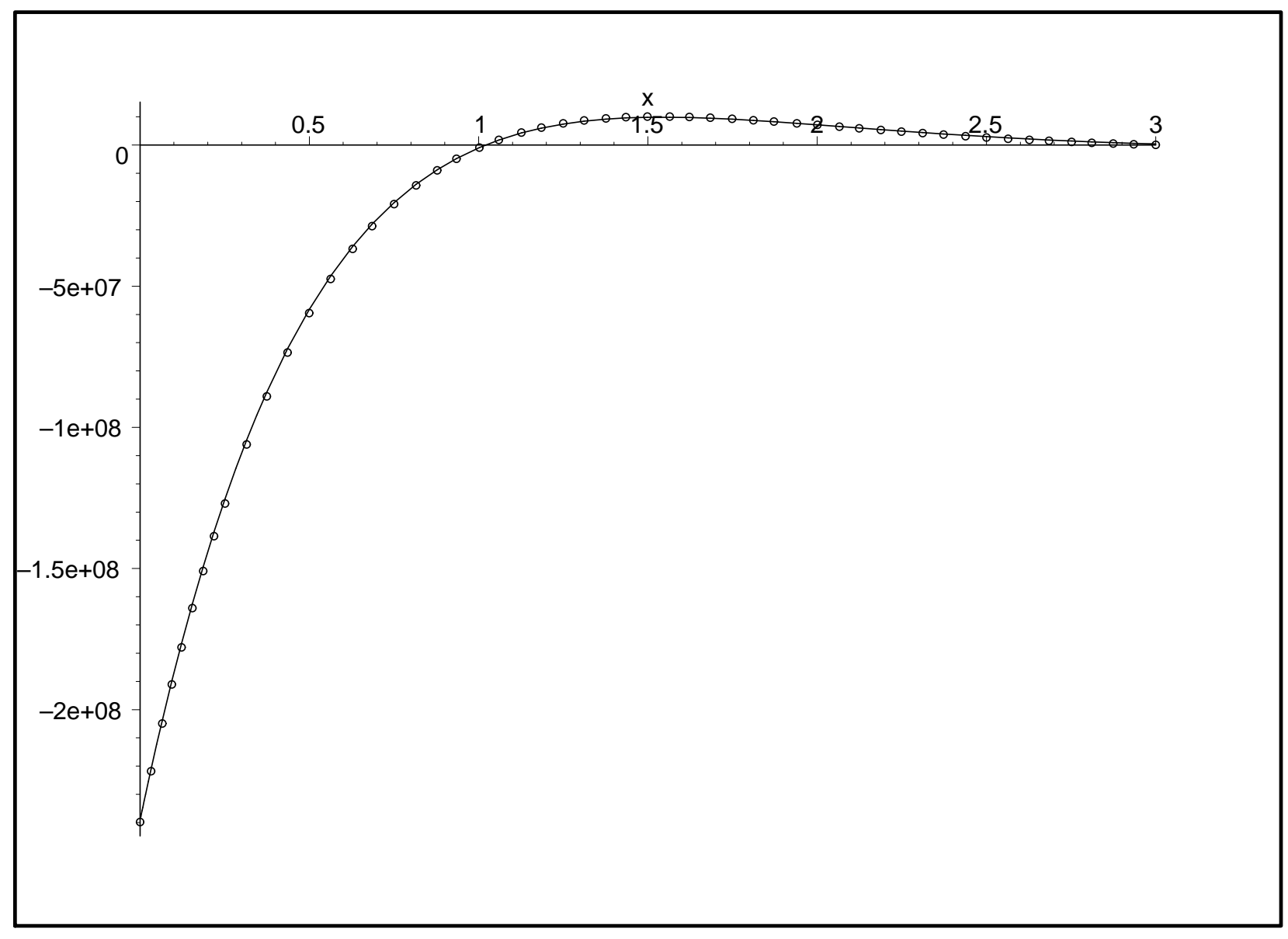

Figure 8: A comparison of $K_{n}(x)$ (solid curve) and $K^{(6)}(x, u)$ (ooo) for $n=25$ with $\varepsilon=0.01$ and $q=0.74894783$. Note that with this choice of parameters, $u=0.024265$.

6. Region VI: $x=O(1), z \approx p, z=p-u \sqrt{p q \varepsilon}, u=O(1)$.

$$
\begin{aligned}
K_{n}(x) & \sim K^{(6)}(x, u)=\frac{\varepsilon^{1 / 2}}{\sqrt{2 \pi p q}}\left[\sqrt{\frac{q \varepsilon}{p}}\right]^{x} \mathrm{D}_{x}(u) \\
& \times \exp \left[\frac{\pi \mathrm{i} p-q \ln (q)}{\varepsilon}+\frac{u \sqrt{p q} \pi \mathrm{i}-u \sqrt{p q} \ln (q)}{\sqrt{\varepsilon}}-\frac{u^{2}}{4}\right],
\end{aligned}
$$

where $\mathrm{D}_{x}(u)$ is the parabolic cylinder function (see Figure 8).

7. Region VII: $0 \ll y<Y^{-}(z), p<z<1$.

$$
K_{n}(x) \sim K^{(7)}(y, z)=\exp \left(\frac{\pi \mathrm{i} y}{\varepsilon}\right)\left[\cos \left(\frac{\pi y}{\varepsilon}\right) K^{+}(y, z)+2 \mathrm{i} \sin \left(\frac{\pi y}{\varepsilon}\right) K^{-}(y, z)\right]
$$

(see Figure 9). 


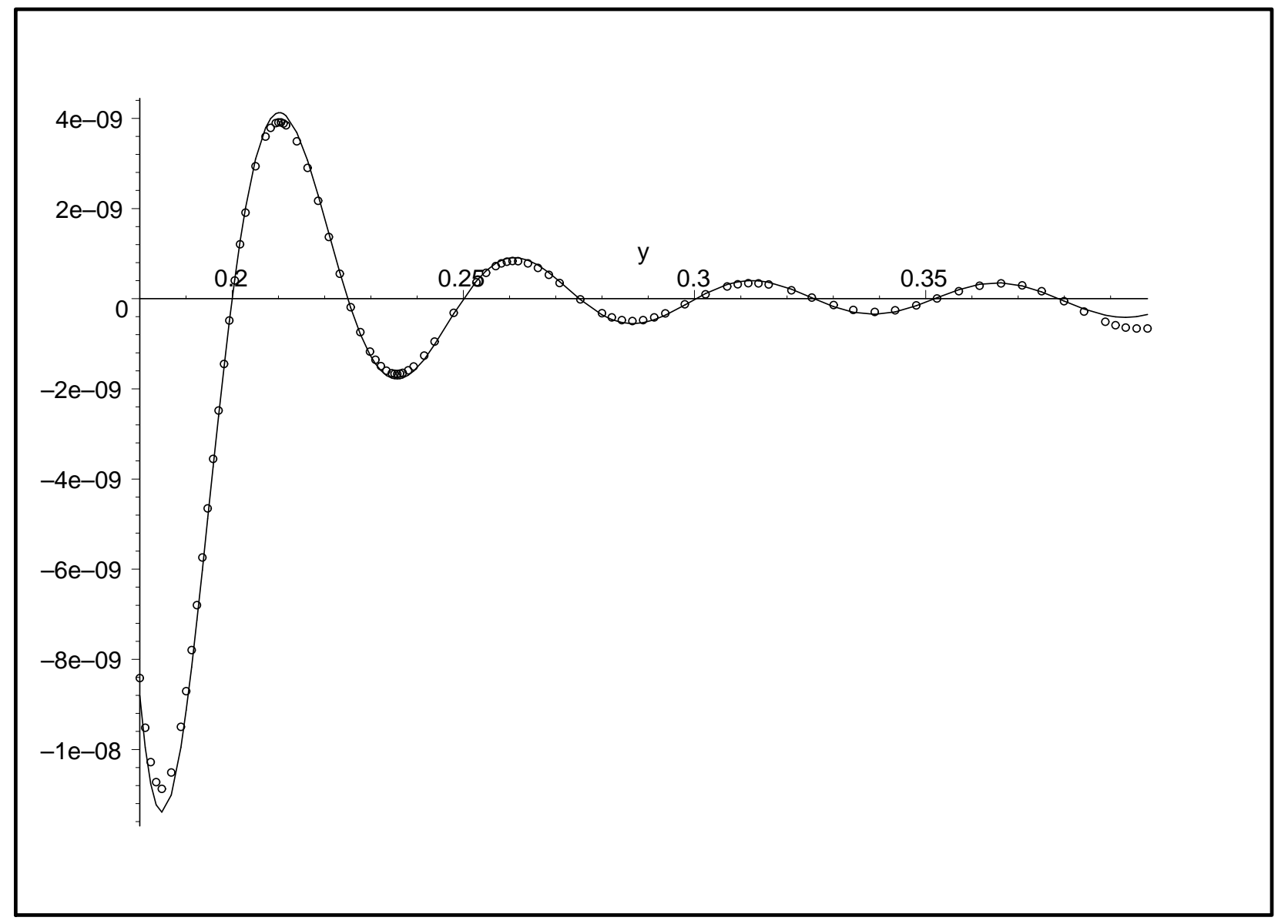

Figure 9: A comparison of $K_{n}(x)$ (solid curve) and $K^{(7)}(y, z)$ (ooo) for $n=35$ with $\varepsilon=0.025$ and $q=0.74894783$. 


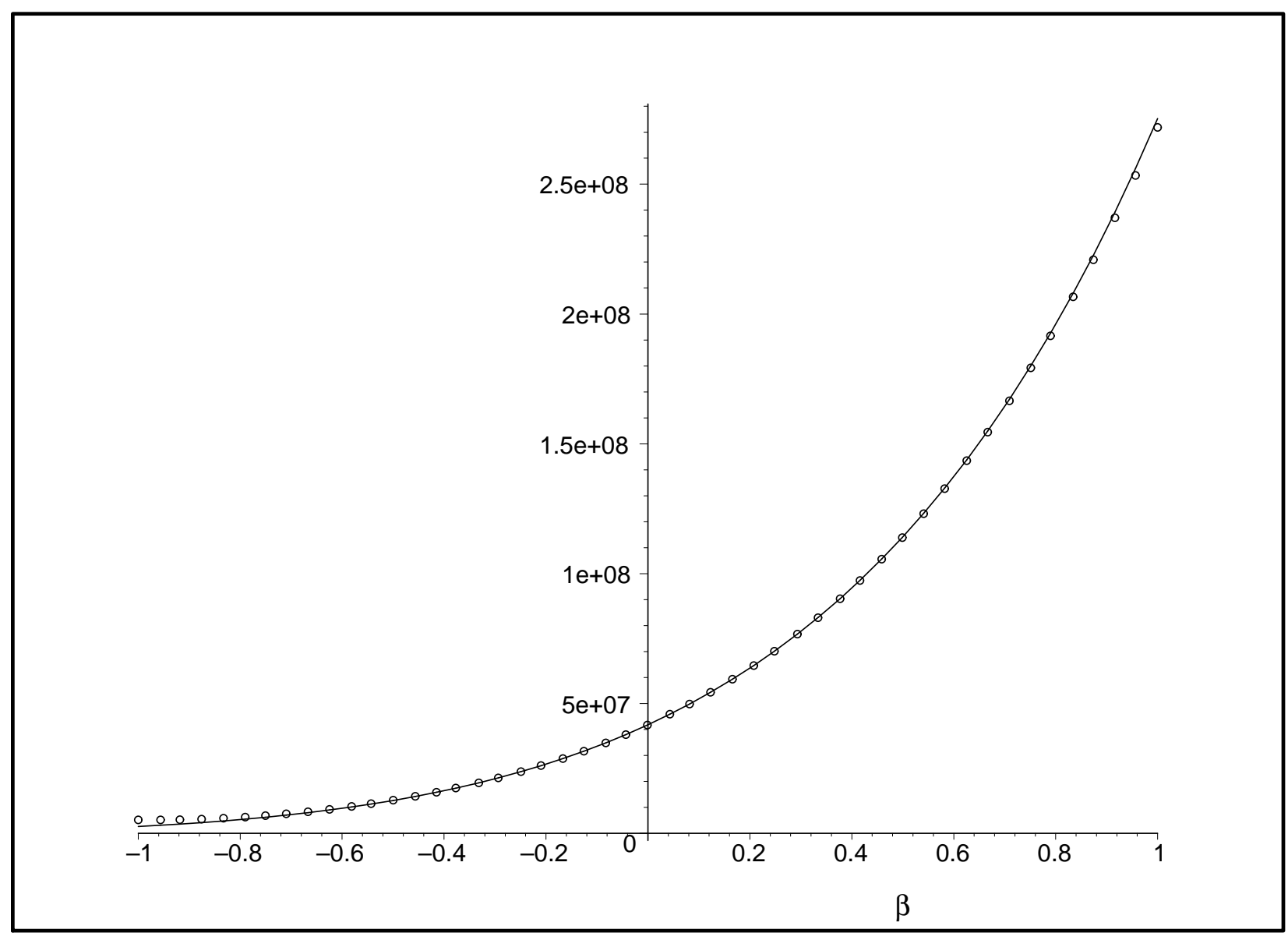

Figure 10: A comparison of $K_{n}(x)$ (solid curve) and $K^{(8)}(\beta, z)$ (ooo) for $n=10$ with $\varepsilon=0.01$ and $q=0.34894783$.

8. Region VIII: $y \approx Y^{-}(z), 0<z<p, y=Y^{-}(z)-\beta \varepsilon^{2 / 3}, \beta=O(1)$.

$$
K_{n}(x) \sim K^{(8)}(\beta, z)=\varepsilon^{1 / 3} \exp \left[\varepsilon^{-1} \psi_{0}(z)+\ln \left(\frac{U_{0}+p}{U_{0}-q}\right) \beta \varepsilon^{-1 / 3}\right] \operatorname{Ai}\left[\Theta^{2 / 3} \beta\right] \frac{\Theta^{-1 / 3}}{\sqrt{z U_{0}}},
$$

where

$$
\begin{gathered}
\psi_{0}(z)=z \pi \mathrm{i}+(z-1) \ln \left(U_{0}\right)+Y^{-}(z) \ln \left(U_{0}-q\right)+\left[1-Y^{-}(z)\right] \ln \left(U_{0}+p\right), \\
\Theta(z)=\sqrt{\frac{U_{0}}{z}} \frac{1}{\left(U_{0}+p\right)\left(U_{0}-q\right)}
\end{gathered}
$$

and $\mathrm{Ai}(\cdot)$ is the Airy function (see Figure [10). 
9. Region IX: $y \approx Y^{-}(z), p<z<1$.

$$
\begin{aligned}
K_{n}(x) & \sim K^{(9)}(\beta, z)=\varepsilon^{1 / 3} \exp \left[\varepsilon^{-1} \psi_{0}(z)+\ln \left(\frac{U_{0}+p}{U_{0}-q}\right) \beta \varepsilon^{-1 / 3}\right] \\
& \times \frac{1}{2} \frac{\vartheta^{-1 / 3}}{\sqrt{z U_{0}}}\left[\lambda^{+}(\beta, z) \operatorname{Ai}\left(\vartheta^{2 / 3} \beta\right)+\mathrm{i} \lambda^{-}(\beta, z) \operatorname{Bi}\left(\vartheta^{2 / 3} \beta\right)\right],
\end{aligned}
$$

where $\vartheta(z)=-\Theta(z)$,

$$
\lambda^{ \pm}(\beta, z)=\exp \left\{\frac{2 \pi \mathrm{i}\left[Y^{-}(z)-\beta \varepsilon^{2 / 3}\right]}{\varepsilon}\right\} \pm 1 .
$$

and $\mathrm{Ai}(\cdot), \mathrm{Bi}(\cdot)$ are the Airy functions (see Figure [11).

10. Region X: $Y^{-}(z)<y<Y^{+}(z), 0<z<1$.

$$
K_{n}(x) \sim K^{(10)}(y, z)=K^{+}(y, z)+K^{-}(y, z)
$$

(see Figure 12).

11. Region XI: $n \approx N, n=N-j, j \in \mathbb{Z}, 0<y<1, y \not \approx q$.

$$
K_{n}(x) \sim K_{j}^{(11)}(y)=\left(\begin{array}{c}
N \\
j
\end{array}\right)(-p)^{N-j}\left(\frac{q}{p}\right)^{N y} \cos (N \pi y)\left(1-\frac{y}{q}\right)^{j}+\left(\begin{array}{c}
N y \\
N-j
\end{array}\right)\left(\frac{1-y}{q-y}\right)^{j+1}
$$

(see Figure 13).

12. Region XII: $n \approx N, y \approx q, y=q+\xi \sqrt{2 p q \varepsilon}, \xi=O(1)$.

$$
\begin{gathered}
K_{n}(x) \sim K_{j}^{(12)}(\xi)=\exp \left[\frac{p \ln (p)+q \ln (q)}{\varepsilon}+\xi \sqrt{\frac{2 p q}{\varepsilon}} \ln \left(\frac{q}{p}\right)-\frac{j}{2} \ln (p q \varepsilon)\right] \exp \left(\frac{\xi^{2}}{2}\right) \\
\times\left[\frac{1}{j !} \mathrm{D}_{j}(\sqrt{2} \xi) \cos \left(\frac{p \pi}{\varepsilon}-\xi \pi \sqrt{\frac{2 p q}{\varepsilon}}\right)-\frac{1}{\sqrt{2 \pi}} \Lambda_{j}(\xi) \sin \left(\frac{p \pi}{\varepsilon}-\xi \pi \sqrt{\frac{2 p q}{\varepsilon}}\right)\right],
\end{gathered}
$$

where the function $\Lambda_{j}: \mathbb{R} \rightarrow \mathbb{R}$ is defined by

$$
\Lambda_{j}(\xi)=\mathrm{i}^{j+1}\left[\mathrm{D}_{-j-1}(\sqrt{2} \mathrm{i} \xi)+(-1)^{j+1} \mathrm{D}_{-j-1}(-\sqrt{2} \mathrm{i} \xi)\right]
$$

(see Figure 14). 


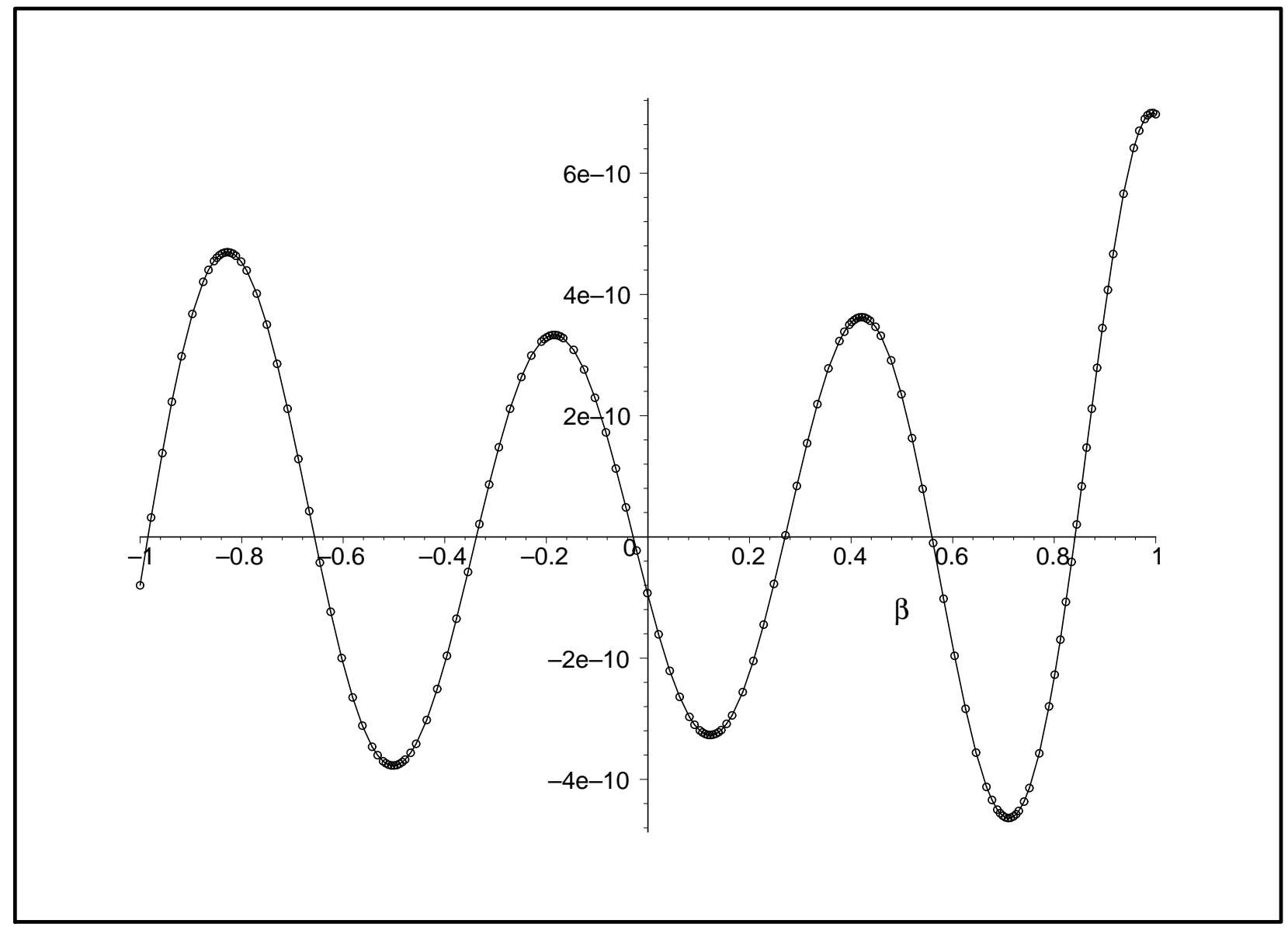

Figure 11: A comparison of $K_{n}(x)$ (solid curve) and $K^{(9)}(\beta, z)$ (ooo) for $n=40$ with $\varepsilon=0.02$ and $q=0.74894783$. 


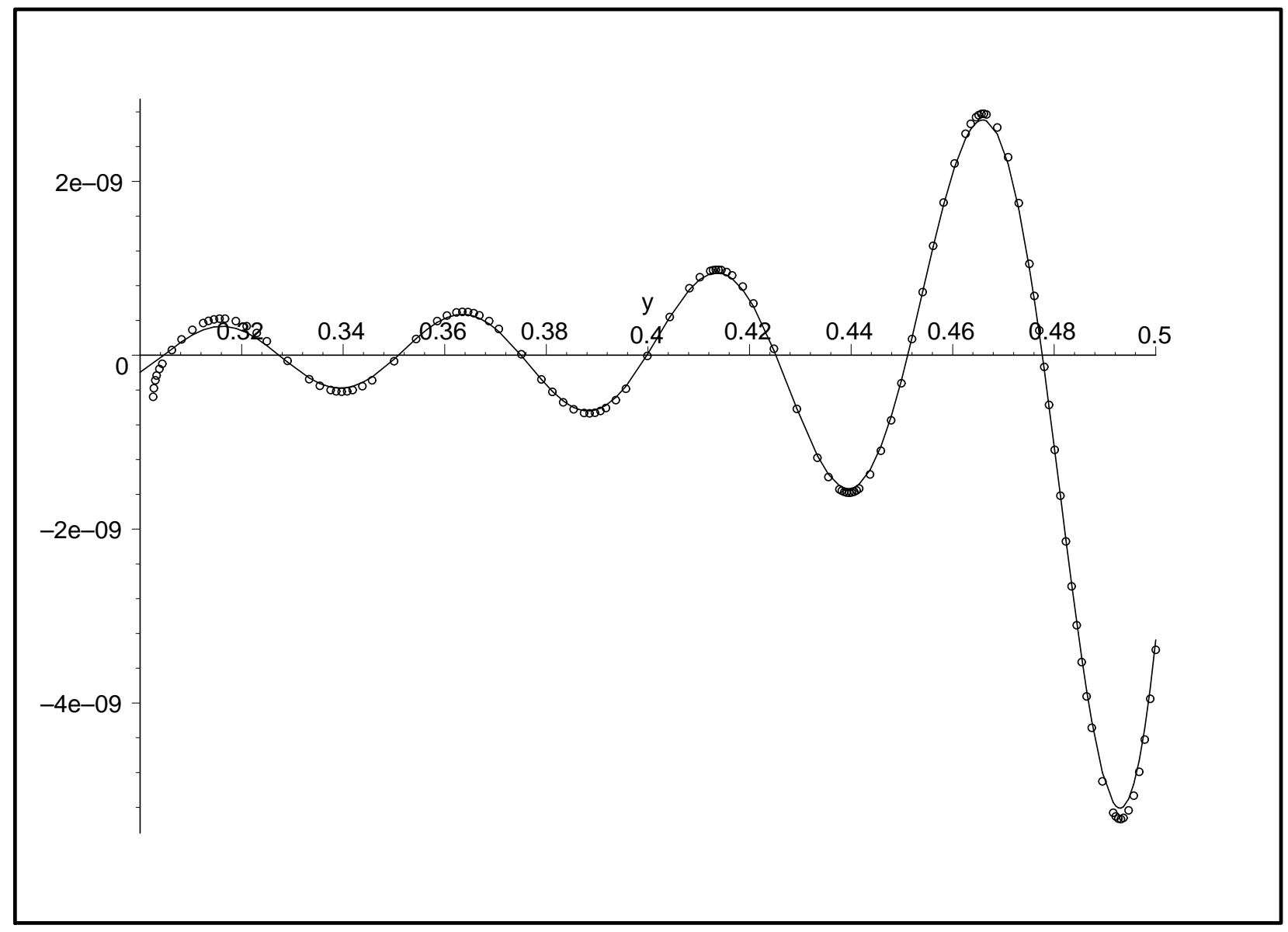

Figure 12: A comparison of $K_{n}(x)$ (solid curve) and $K^{(10)}(y, z)$ (ooo) for $n=40$ with $\varepsilon=0.02$ and $q=0.74894783$. 


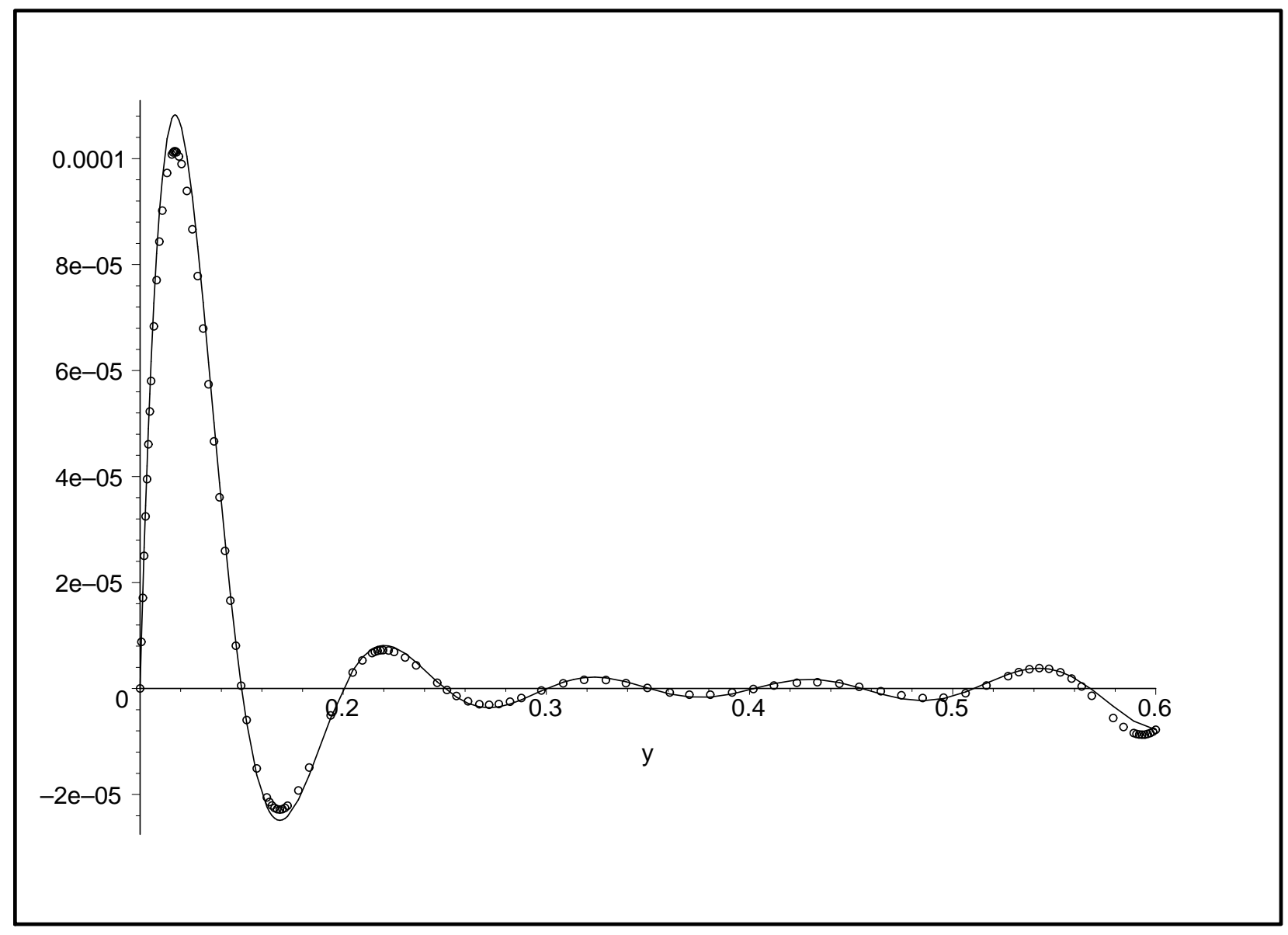

Figure 13: A comparison of $K_{n}(x)$ (solid curve) and $K_{j}^{(11)}(y)$ (ooo) for $n=19$ with $\varepsilon=0.05$ and $q=0.74894783$. 


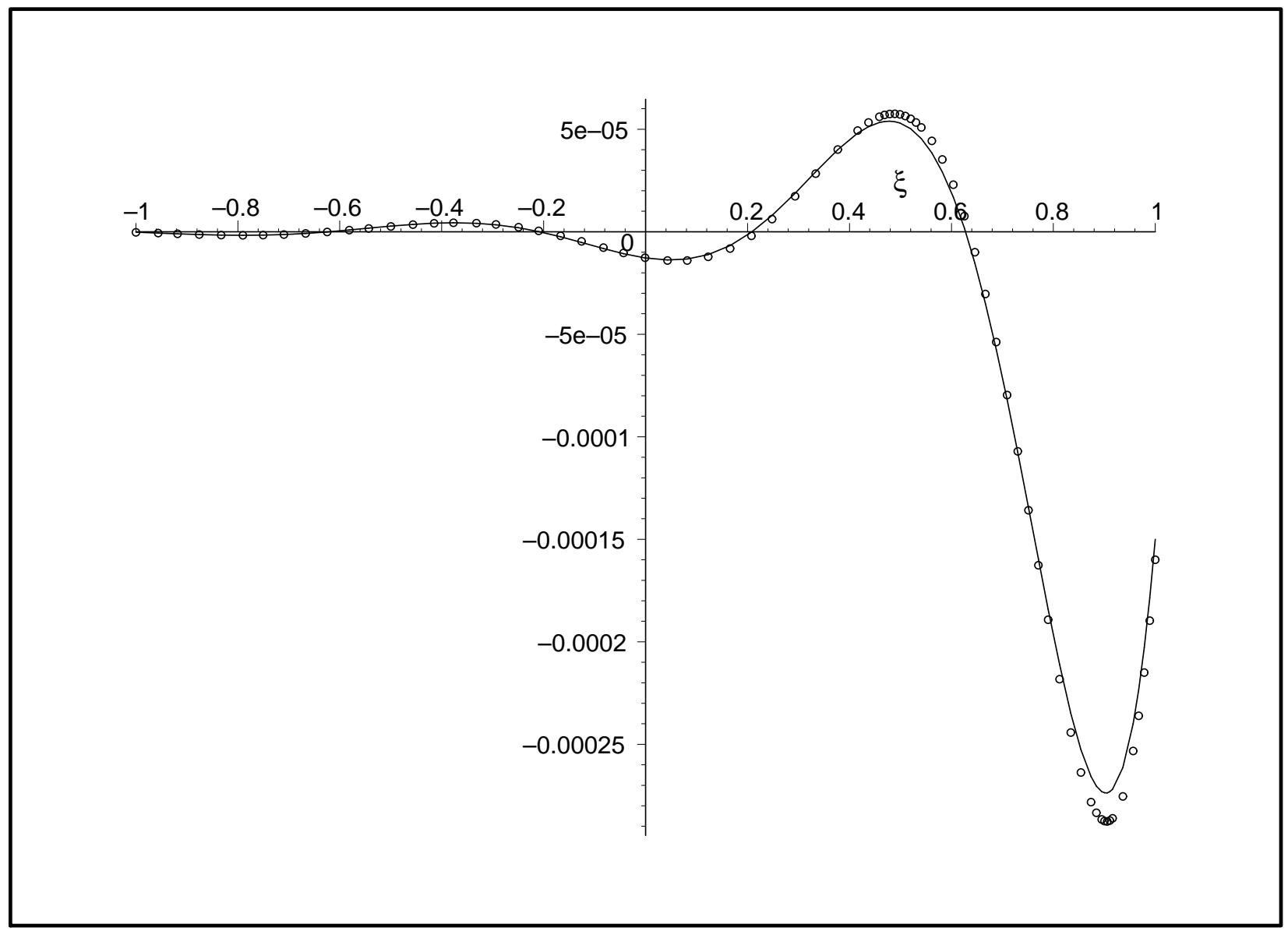

Figure 14: A comparison of $K_{n}(x)$ (solid curve) and $K_{j}^{(12)}(\xi)$ (ooo) for $n=20$ with $\varepsilon=0.05$ and $q=0.74894783$. 


\section{References}

[1] M. Abramowitz and I. A. Stegun, editors. Handbook of mathematical functions with formulas, graphs, and mathematical tables. Dover Publications Inc., New York, 1992. Reprint of the 1972 edition.

[2] N. M. Atakishiyev, G. S. Pogosyan, L. E. Vicent, and K. B. Wolf. Separation of discrete variables in the 2-dim finite oscillator. In Quantum theory and symmetries (Kraków, 2001), pages 255-260. World Sci. Publishing, River Edge, NJ, 2002.

[3] L. A. Bassalygo. Generalization of Lloyd's theorem to arbitrary alphabet. Problems of Control and Information Theory/Problemy Upravlenija i Teorii Informacii, 2(2):133137, 1973.

[4] L. Chihara and D. Stanton. Zeros of generalized Krawtchouk polynomials. J. Approx. Theory, 60(1):43-57, 1990.

[5] P. Delsarte. An algebraic approach to the association schemes of coding theory. Philips Res. Rep. Suppl., (10):vi+97, 1973.

[6] D. Dominici and C. Knessl. Asymptotic analysis by the saddle point method of a datahandling system with many sources. The Journal of Applied Mathematics and Stochastic Analysis. To appear.

[7] P. D. Dragnev and E. B. Saff. A problem in potential theory and zero asymptotics of Krawtchouk polynomials. J. Approx. Theory, 102(1):120-140, 2000.

[8] P. Feinsilver and R. Schott. Krawtchouk polynomials and finite probability theory. In Probability measures on groups, X (Oberwolfach, 1990), pages 129-135. Plenum, New York, 1991.

[9] L. Habsieger. Integer zeros of $q$-Krawtchouk polynomials in classical combinatorics. Adv. in Appl. Math., 27(2-3):427-437, 2001. Special issue in honor of Dominique Foata's 65th birthday (Philadelphia, PA, 2000).

[10] L. Habsieger. Integral zeroes of Krawtchouk polynomials. In Codes and association schemes (Piscataway, NJ, 1999), volume 56 of DIMACS Ser. Discrete Math. Theoret. Comput. Sci., pages 151-165. Amer. Math. Soc., Providence, RI, 2001.

[11] L. Habsieger and D. Stanton. More zeros of Krawtchouk polynomials. Graphs Combin., 9(2):163-172, 1993.

[12] M. E. H. Ismail and P. Simeonov. Strong asymptotics for Krawtchouk polynomials. $J$. Comput. Appl. Math., 100(2):121-144, 1998. 
[13] C. Ivan. A multidimensional nonlinear growth, birth and death, emigration and immigration process. In Proceedings of the Fourth Conference on Probability Theory (Braşov, 1971), pages 421-427. Editura Acad. R. S. R., Bucharest, 1973.

[14] R. Koekoek and R. F. Swarttouw. The Askey-scheme of hypergeometric orthogonal polynomials and its $q$-analogue. Technical Report 98-17, Delft University of Technology, 1998. http://aw.twi.tudelft.nl/ koekoek/askey/.

[15] I. Krasikov. Bounds for the Christoffel-Darboux kernel of the binary Krawtchouk polynomials. In Codes and association schemes (Piscataway, NJ, 1999), volume 56 of DIMACS Ser. Discrete Math. Theoret. Comput. Sci., pages 193-198. Amer. Math. Soc., Providence, RI, 2001.

[16] I. Krasikov and S. Litsyn. On integral zeros of Krawtchouk polynomials. J. Combin. Theory Ser. A, 74(1):71-99, 1996.

[17] I. Krasikov and S. Litsyn. On the distance distributions of BCH codes and their duals. Des. Codes Cryptogr., 23(2):223-231, 2001.

[18] I. Krasikov and S. Litsyn. Survey of binary Krawtchouk polynomials. In Codes and association schemes (Piscataway, NJ, 1999), volume 56 of DIMACS Ser. Discrete Math. Theoret. Comput. Sci., pages 199-211. Amer. Math. Soc., Providence, RI, 2001.

[19] N. N. Lebedev. Special functions and their applications. Dover Publications Inc., New York, 1972. Translated from the Russian.

[20] H. W. Lenstra, Jr. Two theorems on perfect codes. Discrete Math., 3:125-132, 1972.

[21] V. I. Levenshtein. Krawtchouk polynomials and universal bounds for codes and designs in Hamming spaces. IEEE Trans. Inform. Theory, 41(5):1303-1321, 1995.

[22] X.-C. Li and R. Wong. A uniform asymptotic expansion for Krawtchouk polynomials. J. Approx. Theory, 106(1):155-184, 2000.

[23] S. P. Lloyd. Binary block coding. Bell System Tech. J., 36:517-535, 1957.

[24] M. Lorente. Quantum mechanics on discrete space and time. In New developments on fundamental problems in quantum physics (Oviedo, 1996), volume 81 of Fund. Theories Phys., pages 213-224. Kluwer Acad. Publ., Dordrecht, 1997.

[25] F. J. MacWilliams and N. J. A. Sloane. The theory of error-correcting codes. I. NorthHolland Publishing Co., Amsterdam, 1977. North-Holland Mathematical Library, Vol. 16. 
[26] P. Nevai. Two of my favorite ways of obtaining asymptotics for orthogonal polynomials. In Anniversary volume on approximation theory and functional analysis (Oberwolfach, 1983), volume 65 of Internat. Schriftenreihe Numer. Math., pages 417-436. Birkhäuser, Basel, 1984.

[27] A. F. Nikiforov, S. K. Suslov, and V. B. Uvarov. Classical orthogonal polynomials of a discrete variable. Springer Series in Computational Physics. Springer-Verlag, Berlin, 1991. Translated from the Russian.

[28] F. W. J. Olver. Asymptotics and special functions. AKP Classics. A K Peters Ltd., Wellesley, MA, 1997. Reprint of the 1974 original.

[29] A. Poli and L. Huguet. Error correcting codes. Prentice Hall International, Hemel Hempstead, 1992. Translated from the 1989 French original.

[30] W.-Y. Qiu and R. Wong. Asymptotic expansion of the Krawtchouk polynomials and their zeros. Comput. Methods Funct. Theory, 4(1):189-226, 2004.

[31] W. Schoutens. Stochastic processes and orthogonal polynomials, volume 146 of Lecture Notes in Statistics. Springer-Verlag, New York, 2000.

[32] I. I. Sharapudinov. Asymptotic properties of Krawtchouk polynomials. Mat. Zametki, 44(5):682-693, 703, 1988.

[33] N. J. A. Sloane. An introduction to association schemes and coding theory. In Theory and application of special functions (Proc. Advanced Sem., Math. Res. Center, Univ. Wisconsin, Madison, Wis., 1975), pages 225-260. Math. Res. Center, Univ. Wisconsin, Publ. No. 35. Academic Press, New York, 1975.

[34] P. Solé. An inversion formula for Krawtchouk polynomials with applications to coding theory. J. Inform. Optim. Sci., 11(2):207-213, 1990.

[35] R. J. Stroeker and B. M. M. de Weger. On integral zeroes of binary Krawtchouk polynomials. Nieuw Arch. Wisk. (4), 17(2):175-186, 1999.

[36] G. Szegő. Orthogonal polynomials. American Mathematical Society, Providence, R.I., fourth edition, 1975. American Mathematical Society, Colloquium Publications, Vol. XXIII.

[37] Z. Wang and R. Wong. Asymptotic expansions for second-order linear difference equations with a turning point. Numer. Math., 94(1):147-194, 2003.

[38] E. T. Whittaker and G. N. Watson. A course of modern analysis. Cambridge Mathematical Library. Cambridge University Press, Cambridge, 1996. Reprint of the fourth (1927) edition. 
[39] A. Zhedanov. Oscillator 9j-symbols, multidimensional factorization method, and multivariable Krawtchouk polynomials. In Calogero-Moser-Sutherland models (Montréal, QC, 1997), CRM Ser. Math. Phys., pages 549-561. Springer, New York, 2000. 